\title{
Facile Suzuki-Miyaura coupling of activated aryl halides using new CpNiBr(NHC) complexes
}

\author{
Frederick P. Malan, Eric Singleton, Petrus. H. van Rooyen and Marilé Landman* \\ Department of Chemistry, University of Pretoria, 02 Lynnwood Road, Hatfield, Pretoria, 0002, South Africa. \\ *E-mail: marile.landman@up.ac.za
}

\section{Highlights}

- Synthesis of nine new Ni(II)-NHC complexes, [CpNiBr(NHC)].

- Symmetric and asymmetric alkyl/-benzyl/phenylethyl substituted NHC complexes of Ni.

- Suzuki-Miyaura coupling of activated aryl halides with phenylboronic acid.

\section{Graphical abstract}
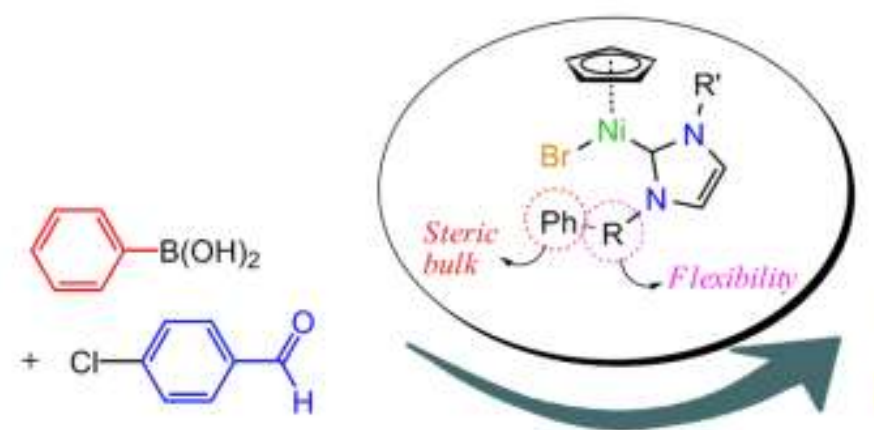

( $3 \mathrm{~mol} \%)$

$\mathrm{K}_{3} \mathrm{PO}_{4}, 1 \mathrm{hr}$

toluene, $90^{\circ} \mathrm{C}$
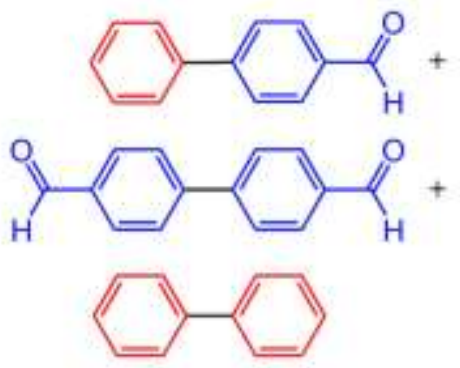

\begin{abstract}
Nine new $\mathrm{Ni}(\mathrm{II})-\mathrm{NHC}$ complexes, [CpNiBr(NHC)], were synthesised from nickelocene and the corresponding symmetric or asymmetric alkyl/-benzyl/phenylethyl imidazolium bromide ligands in relatively high yield. Access to each of the synthesised symmetric or asymmetric alkyl/benzyl/phenylethyl imidazolium bromide salts was obtained through deprotonation of imidazole, followed by treatment with an alkyl- or aryl halide, which is subsequently followed with reaction of a secondary alkyl-, benzyl-, or phenylethyl halide. The series of $[\mathrm{CpNiBr}(\mathrm{NHC})]$ exhibited catalytic activity in the Suzuki-Miyaura coupling of activated aryl halides with phenylboronic acid to give the respective biphenyl and biphenyl-containing products. In general, the more electron-donating NHC-bearing Ni complexes showed higher
\end{abstract}


activity with aryl halides bearing electron-withdrawing functionalities including carboxaldehyde moieties. All complexes were characterised by ${ }^{1} \mathrm{H}-$ and ${ }^{13} \mathrm{C}-\mathrm{NMR}$ spectroscopy, FT-IR spectroscopy, CHN and MS analyses, along with six selected single crystal X-ray structures that are reported here.

Keywords: Nickel; N-heterocyclic carbene; Suzuki-Miyaura coupling

\section{Introduction}

$\mathrm{N}-\mathrm{Heterocyclic}$ carbenes (NHCs) as a class of metal-stabilising "wonder ligands" remain the ligands of choice in many organometallic complexes, with specific application in homogeneous catalysis, organocatalysis, and medicinal chemistry [1-4]. This is mainly due to the support that NHCs provide through the possibility of reliable, predictable, and extensive steric- and electronic tuning $[2,3 b, 5-7]$ in the design of a model complex with the specific application in mind. With the ever-increasing number of transition metal-NHCs reported, NHC-complexes of $\mathrm{Rh}[3 \mathrm{a}, 8], \mathrm{Ru}[9], \mathrm{Ni}$ [7,10-14], Pd [4a,6b,15,16], Ag [17], and $\mathrm{Au}$ $[17,18]$ remain to be the most abundant in literature, noting that Ni-NHC complexes received considerable attention only during the last decade $[11,13,14]$. The reaction of nickelocene with bis(alkyl/aryl)imidazolium halides to yield the complexes [CpNiX(NHC)] $(\mathrm{X}=\mathrm{Cl}, \mathrm{Br}$, I), represents one of the most frequently employed and facile routes into cyclopentadienyl nickel(II) NHC systems [7,11-13,19,21-22]. Since the discovery of this atom-economical reaction by Cowley et al. [23] in 2000 , the series of substituted $\left[\left(\eta^{5}-\mathrm{C}_{5} \mathrm{R}_{5}\right) \mathrm{NiX}(\mathrm{NHC})\right](\mathrm{R}=$ $\mathrm{H}, \mathrm{Me} ; \mathrm{X}=\mathrm{Cl}, \mathrm{Br}, \mathrm{I}, \mathrm{SPh}$ ) complexes has expanded to constitute a relatively well studied class of $\mathrm{Ni}(\mathrm{II}) \mathrm{NHC}$ complexes, predominantly employed in synthetic and catalytic applications [7,11-13,19,21-22].

In homogeneous catalysis, Ni-NHCs occupy an important position in carbon-carbon and carbon-heteroatom organic transformations offering access to an impressive array of valuable molecules which could previously be obtained only via expensive $\mathrm{Ru}$ and $\mathrm{Pd}$ catalysts $[10,11 b, 12,15,19,20,24,25]$. In the plethora of C-C coupling reactions, the Suzuki-Miyaura reaction has become one of the most studied for catalytic applications due to its tolerance of functional groups and low toxicity of its by-products [4d,15,19,20a,25,26]. Furthermore, [CpNiX(NHC)] $(\mathrm{X}=$ halide $)$ and related systems have provided equal or improved catalytic activity in some $\mathrm{C}-\mathrm{C}$ coupling reactions when compared to the traditional $\mathrm{Pd}$ catalysts 
$[10,12,20,25,27]$. In spite of this, the bromo complexes in the series of [CpNiBr(NHC)] compounds reported are few when compared to their chloro- and iodo-analogues $[10,19]$. The known $[\mathrm{CpNiBr}(\mathrm{NHC})]$ complexes $(\mathrm{NHC}=$ imidazolium backbone) reported to date have been illustrated in Figure 1.

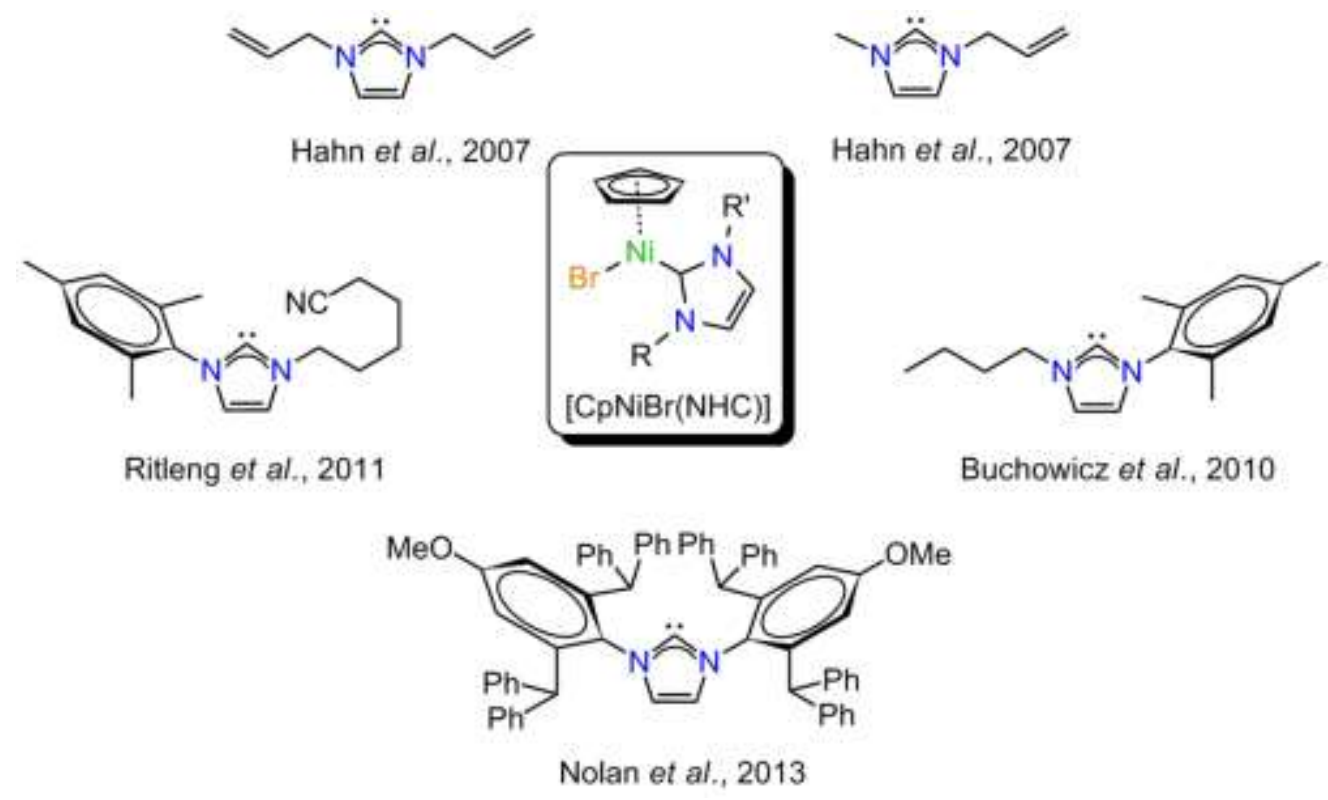

Figure 1: Known [CpNiBr(NHC)] complexes reported by various groups [5a,11e,12,21,22b].

In this study, we expand the existing bromide series by reporting nine novel [ $\mathrm{CpNiBr}(\mathrm{NHC})]$ complexes, where both symmetric- and asymmetric NHC ligands are employed. All new complexes synthesised were characterised, and their catalytic activity evaluated in the Suzuki-Miyaura (SM) coupling reaction using activated aryl chlorides and bromides. One of these substrates, 4-chlorobenzaldehyde, bearing an electron-withdrawing functional group, has to date not been fully investigated previously as a substrate in $[\mathrm{CpNiX}(\mathrm{NHC})](\mathrm{X}=$ halide) catalysed SM reactions.

\section{Material and Methods}

\section{$2.1 \quad$ General}

All experiments were carried out under an argon atmosphere using standard Schlenk techniques. Solvents were dried prior to use using standard techniques [28]. Column chromatography was carried out under inert argon atmospheres using silica gel (particle size 0.063-0.200 $\mathrm{mm}$ ) as the stationary phase. The imidazolium bromide ligands L1 - L9 (Figure 
2) with general formula $[\mathrm{HImRR}] \mathrm{Br}$ with $\mathrm{R}, \mathrm{R}^{\prime}=\mathrm{Me}, \mathrm{Bn},\left(\mathrm{CH}_{2}\right)_{2} \mathrm{Ph}, 4-\mathrm{NO}_{2} \mathrm{Bn}$ were synthesised and purified according to literature procedures [2,13,14,19,21]. NMR characterisation confirmed the molecular structures of the synthesised ligands (see Supplementary Information for data), which correlated with literature data. All other chemicals were purchased from Sigma-Aldrich and used without further purification. ${ }^{1} \mathrm{H}$ (300 $\mathrm{MHz}$ ) and ${ }^{13} \mathrm{C}\{\mathrm{H}\}$ (76 $\left.\mathrm{MHz}\right)$ NMR spectra were recorded on a Bruker ARX-300 spectrometer using either $\mathrm{CDCl}_{3}$, or $\left(\mathrm{CD}_{3}\right)_{2} \mathrm{CO}$ solutions. All measurements were performed at ambient temperature ( $296 \mathrm{~K})$, unless otherwise noted. Chemical shifts were referenced to the internal residual protio solvent impurity at $\delta_{\mathrm{H}} 7.24\left(\mathrm{CDCl}_{3}\right)$ or $2.04\left(\left(\mathrm{CD}_{3}\right)_{2} \mathrm{CO}\right)$; or carbon signals at $\delta_{\mathrm{C}} 77.0\left(\mathrm{CDCl}_{3}\right)$, or 29.8 and $206.3 \mathrm{ppm}\left(\left(\mathrm{CD}_{3}\right)_{2} \mathrm{CO}\right)$. Solid state FT-IR experiments were carried out on a Perkin Elmer Spectrum RXI FT-IR spectrometer as pressed $\mathrm{KBr}$ pellets in air. Microanalytical analyses (\%CHNS) were obtained using a Thermo Scientific Flash 2000 elemental analyzer fitted with a TCD detector. All GC/MS analyses were carried out on a Hewlett Packard (HP) GC 1530A coupled to an Agilent 5975C mass selective detector (MSD). MS (ESI) and MS/MS measurements were performed on a Waters SYNAPT G2 LC-MS spectrometer.

\subsection{General synthesis of [CpNiBr(NHC)] complexes (1-9)}

A suspension of the alkyl/benzyl/phenylethyl imidazolium bromide ( $3 \mathrm{mmol})$ in THF (10 $\mathrm{mL})$ with $\left[\mathrm{Ni}\left(\mathrm{C}_{5} \mathrm{H}_{5}\right)_{2}\right](0.57 \mathrm{~g}, 3 \mathrm{mmol})$ was heated under reflux between $3-16$ hours (depending on the NHC ligand). The reaction mixture was concentrated in vacuo, and purified by silica gel column chromatography, using gradient elution with hexane and dichloromethane. Red to red-brown powders were obtained in relatively high yields.

[CpNiBr\{Im(Bn $\left.\left.)_{2}\right\}\right]$ (1): Yield: 79\%. IR $\left(\mathrm{cm}^{-1}\right): 3168(v(=\mathrm{CH}), \mathrm{w}), 3136(v(=\mathrm{CH}), \mathrm{w}), 3110$ $(v(=\mathrm{CH}), \mathrm{w}), 3028$ ( $v(-\mathrm{CH}), \mathrm{w}), 2929(v(-\mathrm{CH}), \mathrm{w}), 1569$ ( $(v(=\mathrm{CH}), \mathrm{w}), 1495(\delta(-\mathrm{CH}), \mathrm{sym}, \mathrm{m})$, $1453(\delta(-\mathrm{CH})$, sym, s), $1430(\delta(-\mathrm{CH})$, asym, m), $1404(\delta(-\mathrm{CH})$, asym, s), $1358(\delta(-\mathrm{CH}), \mathrm{s})$, $1230(v(-\mathrm{CN}), \mathrm{s}), 1028(v(\mathrm{CN}), \mathrm{w}), 794(\delta(=\mathrm{CH}), \mathrm{s}), 755(\mathrm{~s}), 725(\delta(-\mathrm{CH}), \mathrm{s}), 715(\mathrm{~s}), 685(\mathrm{~s})$. ${ }^{1} \mathrm{H}-\mathrm{NMR}\left(\mathrm{CDCl}_{3}, \delta_{\mathrm{H}}\right) 5.12\left(\mathrm{~s}, \mathrm{C}_{5} H_{5}, 5 \mathrm{H}\right), 6.09\left(\mathrm{dd},{ }^{2} J_{\mathrm{HH}}=15\right.$ and $\left.65 \mathrm{~Hz}, \mathrm{CH}_{2}, 4 \mathrm{H}\right), 6.79$ (s, $\mathrm{NCH}, 2 \mathrm{H}), 7.26-7.40\left(\mathrm{~m}, \mathrm{C}_{6} \mathrm{H}_{5}, 10 \mathrm{H}\right) .{ }^{13} \mathrm{C}\left\{{ }^{1} \mathrm{H}\right\}-\mathrm{NMR}\left(\mathrm{CDCl}_{3}, \delta_{\mathrm{C}}\right) 55.6\left(\mathrm{~s}, \mathrm{CH}_{2}\right), 91.7(\mathrm{~s}$, $\left.C_{5} \mathrm{H}_{5}\right), 122.8(\mathrm{~s}, \mathrm{NCH}), 127.3\left(\mathrm{~s}, C_{6} \mathrm{H}_{5}\right), 127.6\left(\mathrm{~s}, C_{6} \mathrm{H}_{5}\right), 128.0\left(\mathrm{~s}, C_{6} \mathrm{H}_{5}\right), 128.2\left(\mathrm{~s}, C_{6} \mathrm{H}_{5}\right)$, $128.8\left(\mathrm{~s}, C_{6} \mathrm{H}_{5}\right) 129.2\left(\mathrm{~s}, C_{6} \mathrm{H}_{5}\right), 136.3\left(\mathrm{~s}\right.$, ipso- $\left.C_{6} \mathrm{H}_{5}\right), 166.9(\mathrm{~s}, \mathrm{NCN}) . \mathrm{CHN}(\%)$ : $\left[\mathrm{C}_{22} \mathrm{H}_{21} \mathrm{BrN}_{2} \mathrm{Ni}\right]: \mathrm{C}, 58.16$ (58.46), H, 4.68 (4.68), N, 5.91 (6.20). MS (ESI): m/z 452.02 $(\mathrm{M}+)$. 
[CpNiBr\{Im(Me)(Bn)\}] (2): Yield: 77\%. IR $\left(\mathrm{cm}^{-1}\right): 3168(v(=\mathrm{CH}), \mathrm{w}), 3132(v(=\mathrm{CH}), \mathrm{w})$, $3104(v(=\mathrm{CH}), \mathrm{w}), 3053$ (v(-CH), w), 3029 (v(-CH), w), 2924 (v(-CH), w), $1564(v(=\mathrm{CH}), \mathrm{w})$, $1518(\delta(-\mathrm{CH})$, sym, m), $1495(\delta(-\mathrm{CH})$, sym, m), 1454 ( $\delta(-\mathrm{CH})$, sym, s), $1430(\delta(-\mathrm{CH})$, asym, $\mathrm{m}), 1404(\delta(-\mathrm{CH})$, asym, s), $1340(\delta(-\mathrm{CH}), \mathrm{s}), 1230(v(-\mathrm{CN}), \mathrm{s}), 1076(v(\mathrm{CN}), w), 788$ $(\delta(=\mathrm{CH}), \mathrm{s}), 725(\delta(-\mathrm{CH}), \mathrm{s}), 714(\mathrm{~s}), 685(\mathrm{~s}) .{ }^{1} \mathrm{H}-\mathrm{NMR}\left(\mathrm{CDCl}_{3}, \delta_{\mathrm{H}}\right) 4.25\left(\mathrm{~s}, \mathrm{CH}_{3}, 3 \mathrm{H}\right), 5.16$ $\left(\mathrm{s}, \mathrm{C}_{5} H_{5}, 5 \mathrm{H}\right), 5.99\left(\mathrm{~d},{ }^{2} J_{H H}=49 \mathrm{~Hz}, \mathrm{CH}_{2}, 2 \mathrm{H}\right), 6.82\left(\mathrm{~d},{ }^{3} J_{H H}=48 \mathrm{~Hz}, \mathrm{NCH}, 2 \mathrm{H}\right), 7.20-7.31$ $\left(\mathrm{m}, \mathrm{C}_{6} H_{5}, 5 \mathrm{H}\right) .{ }^{13} \mathrm{C}\left\{{ }^{1} \mathrm{H}\right\}-\mathrm{NMR}\left(\mathrm{CDCl}_{3}, \delta_{\mathrm{C}}\right) 38.8\left(\mathrm{~s}, \mathrm{CH}_{3}\right), 55.1\left(\mathrm{~s}, \mathrm{CH}_{2}\right), 91.2\left(\mathrm{~s}, C_{5} \mathrm{H}_{5}\right), 121.9$ $(\mathrm{s},\{\mathrm{Me}\} \mathrm{NCH}), 123.6(\mathrm{~s},\{\mathrm{Bn}\} \mathrm{NCH}), 127.3\left(\mathrm{~s}, C_{6} \mathrm{H}_{5}\right), 127.6\left(\mathrm{~s}, C_{6} \mathrm{H}_{5}\right), 128.4\left(\mathrm{~s}, C_{6} \mathrm{H}_{5}\right), 136.0$ (s, ipso- $\left.C_{6} \mathrm{H}_{5}\right), 161.8(\mathrm{~s}, \mathrm{NCN}) . \mathrm{CHN}(\%)$ : [ $\left.\mathrm{C}_{16} \mathrm{H}_{17} \mathrm{BrN}_{2} \mathrm{Ni}\right]$ : C, 50.96 (51.12), H, 4.31 (4.56), N, 7.75 (7.45). MS (ESI): m/z $375.99(\mathrm{M}+)$.

[CpNiBr\{Im(Me) $\left.\left.\left(\left(\mathrm{CH}_{2}\right)_{2} \mathrm{Ph}\right)\right\}\right]$ (3): Yield: 74\%. IR $\left(\mathrm{cm}^{-1}\right): 3156(v(=\mathrm{CH}), \mathrm{w}), 3124(v(=\mathrm{CH})$, w), $3100(v(=\mathrm{CH}), \mathrm{w}), 3054(v(-\mathrm{CH}), \mathrm{w}), 2922$ (v(-CH), w), $1604(v(=\mathrm{CH}), \mathrm{s}), 1519(\delta(-\mathrm{CH})$, sym, s), 1492 ( $\delta(-\mathrm{CH})$, sym, m), $1460(\delta(-\mathrm{CH})$, sym, s), 1408 ( $\delta(-\mathrm{CH})$, asym, s), 1339 ( $\delta(-$ $\mathrm{CH}), \mathrm{s}), 1233$ (v(-CN), s), $1081(v(\mathrm{CN}), \mathrm{w}), 786(\delta(=\mathrm{CH}), \mathrm{s}), 727(\delta(-\mathrm{CH}), \mathrm{s}), 714(\mathrm{~s}), 697$ (s). ${ }^{1} \mathrm{H}-\mathrm{NMR}\left(\left\{\mathrm{CD}_{3}\right\}_{2} \mathrm{CO}, \delta_{\mathrm{H}}\right) 3.41\left(\mathrm{br} \mathrm{s}, \mathrm{NCH}_{2}, 2 \mathrm{H}\right), 4.28\left(\mathrm{~s}, \mathrm{CH}_{3}, 3 \mathrm{H}\right), 4.95\left(\mathrm{~d},{ }^{3} J_{H H}=29\right.$ $\left.\mathrm{Hz}, \mathrm{CH}_{2} \mathrm{C}_{6} \mathrm{H}_{5}, 2 \mathrm{H}\right), 5.20$ (s, $\left.\mathrm{C}_{5} H_{5}, 5 \mathrm{H}\right), 7.13$ (s, NCH, 1H), 7.20 (s, $\left.\mathrm{NCH}, 1 \mathrm{H}\right), 7.23-7.38$ $\left(\mathrm{m}, \mathrm{C}_{6} H_{5}, 5 \mathrm{H}\right) .{ }^{13} \mathrm{C}\left\{{ }^{1} \mathrm{H}\right\}-\mathrm{NMR}\left(\left\{\mathrm{CD}_{3}\right\}_{2} \mathrm{CO}, \delta_{\mathrm{C}}\right) 37.7\left(\mathrm{~s}, \mathrm{CH}_{3}\right), 39.1\left(\mathrm{~s}, \mathrm{NCH}_{2}\right), 53.9$ (s, $\left.\mathrm{CH}_{2} \mathrm{C}_{6} \mathrm{H}_{5}\right), 92.0\left(\mathrm{~s}, C_{5} \mathrm{H}_{5}\right), 123.4(\mathrm{~s},\{\mathrm{Me}\} \mathrm{NCH}), 124.5$ (s, $\left.\{\mathrm{PhEt}\} \mathrm{NCH}\right), 127.3\left(\mathrm{~s}, C_{6} \mathrm{H}_{5}\right)$, $127.6\left(\mathrm{~s}, C_{6} \mathrm{H}_{5}\right), 129.3\left(\mathrm{~s}, C_{6} \mathrm{H}_{5}\right), 129.9\left(\mathrm{~s}, C_{6} \mathrm{H}_{5}\right), 139.7$ (s, ipso- $\left.C_{6} \mathrm{H}_{5}\right), 161.3$ (s, NCN). CHN (\%): $\left[\mathrm{C}_{17} \mathrm{H}_{19} \mathrm{BrN}_{2} \mathrm{Ni}\right]: \mathrm{C}, 52.49$ (52.36), H, 4.58 (4.91), N, 6.90 (7.18). MS (ESI): m/z 388.19 $(\mathrm{M}+)$.

[CpNiBr\{Im(Me)(4-NO $\left.\left.\left.\mathrm{I}_{2} \mathrm{Bn}\right)\right\}\right]$ (4): Yield: 69\%. IR $\left(\mathrm{cm}^{-1}\right): 3168(v(=\mathrm{CH}), \mathrm{w}), 3132(v(=\mathrm{CH})$, w), $3109(v(=\mathrm{CH}), w), 3053(v(-\mathrm{CH}), \mathrm{w}), 2929$ (v(-CH), w), 1674 (v(NO), asym, m), 1516 $(\delta(-\mathrm{CH})$, sym, s), 1454 ( $\delta(-\mathrm{CH})$, sym, s), 1430 ( $\delta(-\mathrm{CH})$, asym, m), $1404(\delta(-\mathrm{CH})$, asym, s), $1344(\delta(-\mathrm{CH}), \mathrm{s}), 1230$ ( $(\mathrm{-CN}), \mathrm{s}), 1166$ ( $(\mathrm{NO}), \mathrm{sym}, \mathrm{m}), 1108(\mathrm{~m}), 1013(\mathrm{v}(\mathrm{CN}), \mathrm{w}), 858$ $(\mathrm{m}), 795(\delta(=\mathrm{CH}), \mathrm{s}), 726(\delta(-\mathrm{CH}), \mathrm{s}), 715(\mathrm{~s}), 685(\mathrm{~s}) .{ }^{1} \mathrm{H}-\mathrm{NMR}\left(\mathrm{CDCl}_{3}, \delta_{\mathrm{H}}\right) 4.30\left(\mathrm{~s}, \mathrm{CH}_{3}\right.$, $3 \mathrm{H}), 5.19\left(\mathrm{~s}, \mathrm{C}_{5} H_{5}, 5 \mathrm{H}\right), 6.16\left(\mathrm{~d},{ }^{2} J_{H H}=39 \mathrm{~Hz}, \mathrm{CH}_{2}, 2 \mathrm{H}\right), 6.89\left(\mathrm{~d},{ }^{3} J_{H H}=55 \mathrm{~Hz}, \mathrm{NCH}, 2 \mathrm{H}\right)$, $7.46\left(\mathrm{~s}, \mathrm{C}_{6} H_{4}, 2 \mathrm{H}\right), 8.22\left(\mathrm{~s}, \mathrm{C}_{6} H_{4}\right.$ adjacent to $\left.\mathrm{NO}_{2}, 2 \mathrm{H}\right) .{ }^{13} \mathrm{C}\left\{{ }^{1} \mathrm{H}\right\}-\mathrm{NMR}\left(\mathrm{CDCl}_{3}, \delta_{\mathrm{C}}\right) 25.6(\mathrm{~s}$, $\mathrm{CH}_{3}$ ), $39.3\left(\mathrm{~s}, \mathrm{CH}_{2}\right), 91.8\left(\mathrm{~s}, C_{5} \mathrm{H}_{5}\right), 122.2\left(\mathrm{~s},\{\mathrm{Me}\} \mathrm{NCH}\right.$ ), 124.2 (s, $\left.C_{6} \mathrm{H}_{4}\right), 124.6$ (s, \{4$\left.\left.\mathrm{NO}_{2} \mathrm{Bn}\right\} \mathrm{NCH}\right), 128.6\left(\mathrm{~s}, C_{6} \mathrm{H}_{4}\right.$ adjacent to $\mathrm{NO}_{2}$ ), 143.8 (s, ipso- $\left.C_{6} \mathrm{H}_{4}\right), 147.8$ (s, ipso- $C_{6} \mathrm{H}_{4}$ containing $\mathrm{NO}_{2}$ ), $164.8(\mathrm{~s}, \mathrm{NCN})$. $\mathrm{CHN}(\%)$ : $\left[\mathrm{C}_{16} \mathrm{H}_{16} \mathrm{BrN}_{3} \mathrm{NiO}_{2}\right]: \mathrm{C}, 45.64$ (45.66), H, 3.52 (3.83), N, 9.61 (9.98). MS (ESI): m/z $418.98(\mathrm{M}+)$. 
[CpNiBr $\left.\left\{\operatorname{Im}\left(\left(\mathrm{CH}_{2}\right)_{2} \mathrm{Ph}\right)_{2}\right\}\right]$ (5): Yield: 71\%. IR $\left(\mathrm{cm}^{-1}\right): 3120(v(=\mathrm{CH}), \mathrm{w}), 3101(v(=\mathrm{CH}), \mathrm{w})$, 3048 (v(-CH), w), 2922 (v(-CH), w), $2443(\mathrm{w}), 1675(\mathrm{~m}), 1603(v(=\mathrm{CH}), \mathrm{s}), 1519(\delta(-\mathrm{CH})$, sym, s), 1459 ( $\delta(-\mathrm{CH})$, sym, m), $1441(\delta(-\mathrm{CH})$, sym, s), 1408 ( $\delta(-\mathrm{CH})$, asym, s), 1341 ( $\delta(-$ $\mathrm{CH}), \mathrm{s}), 1234$ (v(-CN), s), $1108(v(\mathrm{CN}), \mathrm{m}), 1013(\mathrm{w}), 859(\mathrm{~s}), 786(\delta(=\mathrm{CH}), \mathrm{s}), 734(\delta(-\mathrm{CH})$, s), $679(\mathrm{~m}) .{ }^{1} \mathrm{H}-\mathrm{NMR}\left(\mathrm{CDCl}_{3}, \delta_{\mathrm{H}}\right) 3.32\left(\mathrm{~m}, \mathrm{NCH}_{2}, 4 \mathrm{H}\right), 4.98\left(\mathrm{~m}, \mathrm{CH}_{2} \mathrm{C}_{6} \mathrm{H}_{5}, 2 \mathrm{H}\right), 5.17$ (s, $\left.\mathrm{C}_{5} H_{5}, 5 \mathrm{H}\right), 6.61(\mathrm{~s}, \mathrm{NCH}, 2 \mathrm{H}), 7.24-7.34\left(\mathrm{~m}, \mathrm{C}_{6} H_{5}, 10 \mathrm{H}\right) .{ }^{13} \mathrm{C}\left\{{ }^{1} \mathrm{H}\right\}-\mathrm{NMR}\left(\mathrm{CDCl}_{3}, \delta_{\mathrm{C}}\right) 37.2$ (s, $\left.\mathrm{NCH}_{2}\right), 53.5\left(\mathrm{~s}, \mathrm{CH}_{2} \mathrm{C}_{6} \mathrm{H}_{5}\right), 91.8\left(\mathrm{~s}, \mathrm{C}_{5} \mathrm{H}_{5}\right), 122.2(\mathrm{~s}, \mathrm{NCH}), 126.8\left(\mathrm{~s}, C_{6} \mathrm{H}_{5}\right), 128.7$ (s, $\left.C_{6} \mathrm{H}_{5}\right), 129.0$ (s, $C_{6} \mathrm{H}_{5}$ ), 138.1 (s, ipso- $C_{6} \mathrm{H}_{5}$ ), 161.3 (s, NCN). CHN (\%): [ $\left.\mathrm{C}_{24} \mathrm{H}_{25} \mathrm{BrN}_{2} \mathrm{Ni}\right]: \mathrm{C}$, 60.21 (60.05), H, 5.38 (5.25), N, 6.13 (5.84). MS (ESI): m/z 480.43 (M+).

[CpNiBr\{Im(Bn)(4-NO$\left.\left.\left.{ }_{2} \mathrm{Bn}\right)\right\}\right]$ (6): Yield: 74\%. IR $\left(\mathrm{cm}^{-1}\right): 3163(v(=\mathrm{CH}), \mathrm{w}), 3090(v(=\mathrm{CH})$, m), $3020(v(-\mathrm{CH}), w), 2921$ (v(-CH), w), $2846(\mathrm{w}), 1674$ (v(NO), asym, m), 1597 (s), 1517 ( $\delta(-\mathrm{CH})$, sym, s), 1496 (s), 1455 ( $\delta(-\mathrm{CH})$, sym, s), 1435 ( $\delta(-\mathrm{CH})$, asym, m), 1407 ( $\delta(-\mathrm{CH})$, asym, s), 1343 ( $\delta(-\mathrm{CH}), \mathrm{s}), 1230(\mathrm{v}(-\mathrm{CN}), \mathrm{s}), 1208$ (v(NO), sym, m), $1108(\mathrm{~m}), 1044(\mathrm{v}(\mathrm{CN})$, w), $1011(\mathrm{~m}), 859(\mathrm{~m}), 838(\mathrm{~m}), 788(\delta(=\mathrm{CH}), \mathrm{s}), 734(\delta(-\mathrm{CH}), \mathrm{s}), 714(\mathrm{~s}), 684(\mathrm{~s}) .{ }^{1} \mathrm{H}-\mathrm{NMR}$ $\left(\mathrm{CDCl}_{3}, \delta_{\mathrm{H}}\right) 5.13\left(\mathrm{~s}, \mathrm{C}_{5} \mathrm{H}_{5}, 5 \mathrm{H}\right), 6.08\left(\mathrm{~m},\{\mathrm{Bn}\} \mathrm{CH}_{2}, 2 \mathrm{H}\right), 6.23\left(\mathrm{~m},\left\{4-\mathrm{NO}_{2} \mathrm{Bn}\right\} \mathrm{CH}_{2}, 2 \mathrm{H}\right), 6.84$ $\left(\mathrm{d},{ }^{3} J_{H H}=9 \mathrm{~Hz}, \mathrm{NCH}, 2 \mathrm{H}\right), 7.28-7.50\left(\mathrm{~m}, \mathrm{C}_{6} H_{4}\right.$ and $\left.\mathrm{C}_{6} H_{5}, 2 \mathrm{H}\right), 8.23$ (s, $\mathrm{C}_{6} H_{4}$ adjacent to $\left.\mathrm{NO}_{2}, 2 \mathrm{H}\right) .{ }^{13} \mathrm{C}\left\{{ }^{1} \mathrm{H}\right\}-\mathrm{NMR}\left(\mathrm{CDCl}_{3}, \delta_{\mathrm{C}}\right) 55.1\left(\mathrm{~s}, \mathrm{CH}_{2} \mathrm{C}_{6} \mathrm{H}_{5}\right), 55.9\left(\mathrm{~s}, \mathrm{CH}_{2} \mathrm{C}_{6} \mathrm{H}_{4} \mathrm{NO}_{2}\right), 91.9$ (s, $\left.C_{5} \mathrm{H}_{5}\right), 122.5(\mathrm{~s},\{\mathrm{Bn}\} \mathrm{NCH}), 123.4\left(\mathrm{~s},\left\{4-\mathrm{NO}_{2} \mathrm{Bn}\right\} \mathrm{NCH}\right), 123.7\left(\mathrm{~s}, C_{6} \mathrm{H}_{5}\right), 124.2\left(\mathrm{~s}, C_{6} \mathrm{H}_{5}\right)$, $127.8\left(\mathrm{~s}, C_{6} \mathrm{H}_{4}\right), 128.4\left(\mathrm{~s}, C_{6} \mathrm{H}_{5}\right), 128.6\left(\mathrm{~s}, C_{6} \mathrm{H}_{5}\right), 129.1\left(\mathrm{~s}, C_{6} \mathrm{H}_{4}\right.$ adjacent to $\left.\mathrm{NO}_{2}\right), 136.0(\mathrm{~s}$, ipso- $C_{6} \mathrm{H}_{5}$ ), 143.7 (s, ipso- $\left.C_{6} \mathrm{H}_{4}\right), 147.8$ (s, ipso- $C_{6} \mathrm{H}_{5}$ containing $\mathrm{NO}_{2}$ ), 165.8 (s, NCN). CHN (\%): $\left[\mathrm{C}_{17} \mathrm{H}_{15} \mathrm{BrN}_{3} \mathrm{NiO}_{2}\right]$ : C, 53.41(53.16), H, 4.19(4.06), N, 8.10(8.45). MS (ESI): m/z 465.08 $(\mathrm{M}+)$.

[CpNiBr\{Im(Bn) $\left.\left.\left(\left(\mathrm{CH}_{2}\right)_{2} \mathrm{Ph}\right)\right\}\right]$ (7): Yield: 73\%. IR $\left(\mathrm{cm}^{-1}\right): 3154(v(=\mathrm{CH}), \mathrm{w}), 3100(\mathrm{v}(=\mathrm{CH})$, w), $3054(v(-\mathrm{CH}), w), 2922(v(-\mathrm{CH}), \mathrm{w}), 2437(\mathrm{w}), 1674(\mathrm{~m}), 1604(v(=\mathrm{CH}), \mathrm{s}), 1518(\delta(-$ $\mathrm{CH})$, sym, s), 1459 ( $\delta(-\mathrm{CH})$, sym, m), 1408 ( $\delta(-\mathrm{CH})$, asym, s), $1340(\delta(-\mathrm{CH}), \mathrm{s}), 1233$ (v($\mathrm{CN}), \mathrm{s}), 1108(\mathrm{v}(\mathrm{CN}), \mathrm{m}), 1014(\mathrm{w}), 859(\mathrm{~m}), 832(\mathrm{~m}), 786(\delta(=\mathrm{CH}), \mathrm{s}), 734(\delta(-\mathrm{CH}), \mathrm{s}), 700$ $(\mathrm{m}), 679(\mathrm{~m}) .{ }^{1} \mathrm{H}-\mathrm{NMR}\left(\mathrm{CDCl}_{3}, \delta_{\mathrm{H}}\right) 3.36\left(\mathrm{q},{ }^{3} J_{H H}=9 \mathrm{~Hz}, \mathrm{NCH}_{2} \mathrm{CH}_{2}, 2 \mathrm{H}\right), 5.00\left(\mathrm{t},{ }^{3} J_{H H}=8\right.$ $\left.\mathrm{Hz}, \mathrm{CH}_{2} \mathrm{C}_{6} \mathrm{H}_{5}, 2 \mathrm{H}\right), 5.12\left(\mathrm{~s}, \mathrm{C}_{5} H_{5}, 5 \mathrm{H}\right), 6.10\left(\mathrm{dd},{ }^{2} J_{H H}=15\right.$ and $\left.116 \mathrm{~Hz}, \mathrm{NCH}_{2} \mathrm{C}_{6} \mathrm{H}_{5}, 2 \mathrm{H}\right)$, $6.74\left(\mathrm{q},{ }^{3} J_{H H}=2 \mathrm{~Hz}, \mathrm{NCH}, 2 \mathrm{H}\right), 7.18-7.39\left(\mathrm{~m}, \mathrm{C}_{6} H_{5}, 10 \mathrm{H}\right) .{ }^{13} \mathrm{C}\left\{{ }^{1} \mathrm{H}\right\}-\mathrm{NMR}\left(\mathrm{CDCl}_{3}, \delta_{\mathrm{C}}\right)$ 37.2 (s, $\mathrm{CH}_{2} \mathrm{CH}_{2} \mathrm{C}_{6} \mathrm{H}_{5}$ ), 53.5 (s, $\mathrm{CH}_{2} \mathrm{CH}_{2} \mathrm{C}_{6} \mathrm{H}_{5}$ ), 55.7 (s, $\mathrm{CH}_{2} \mathrm{C}_{6} \mathrm{H}_{5}$ ), 91.8 (s, $C_{5} \mathrm{H}_{5}$ ), 122.3 (s, $\{\mathrm{PhEt}\} \mathrm{NCH}), 122.7(\mathrm{~s},\{\mathrm{Bn}\} \mathrm{NCH}), 126.9\left(\mathrm{~s}, C_{6} \mathrm{H}_{5}\right), 127.6\left(\mathrm{~s}, C_{6} \mathrm{H}_{5}\right), 128.1\left(\mathrm{~s}, C_{6} \mathrm{H}_{5}\right), 128.7$ 
(s, $\left.C_{6} \mathrm{H}_{5}\right), 128.9\left(\mathrm{~s}, C_{6} \mathrm{H}_{5}\right), 129.0\left(\mathrm{~s}, C_{6} \mathrm{H}_{5}\right), 136.6$ (s, ipso- $C_{6} \mathrm{H}_{5}$ of $\left.\mathrm{PhEt}\right), 138.0$ (s, ipso- $C_{6} \mathrm{H}_{5}$ of $\mathrm{Bn}), 162.9$ (s, NCN). CHN (\%): [ $\left.\mathrm{C}_{23} \mathrm{H}_{23} \mathrm{BrN}_{2} \mathrm{Ni}\right]$ : C, 59.01 (59.28), H, 4.86 (4.97), N, 6.29 (6.01). MS (ESI): m/z $495.45(\mathrm{M}+)$.

[CpNiBr\{Im((CH$\left.\left.\left.)_{2} \mathrm{Ph}\right)\left(4-\mathrm{NO}_{2} \mathrm{Bn}\right)\right\}\right]$ (8): Yield: 70\%. IR $\left(\mathrm{cm}^{-1}\right): 3148(v(=\mathrm{CH}), \mathrm{w}), 3116$ $(v(=\mathrm{CH}), \mathrm{w}), 3109(v(=\mathrm{CH}), \mathrm{w}), 2937(v(-\mathrm{CH}), \mathrm{m}), 2840(v(-\mathrm{CH}), \mathrm{w}), 1674(v(\mathrm{NO})$, asym, m), 1598 (s), $1516(\delta(-\mathrm{CH})$, sym, s), 1453 ( $\delta(-\mathrm{CH})$, sym, s), 1412 ( $\delta(-\mathrm{CH})$, asym, m), 1341 $(\delta(-\mathrm{CH}), \mathrm{s}), 1263(\mathrm{~m}), 1233$ (v(-CN), s), 1183 (v(NO), sym, m), $1108(\mathrm{~m}), 1015$ (v(CN), w), $858(\mathrm{~m}), 833(\mathrm{~m}), 786(\delta(=\mathrm{CH}), \mathrm{s}), 739(\delta(-\mathrm{CH}), \mathrm{s}), 721(\mathrm{~s}), 700(\mathrm{~s}) .{ }^{1} \mathrm{H}-\mathrm{NMR}\left(\mathrm{CDCl}_{3}, \delta_{\mathrm{H}}\right)$ 3.40 (br s, $\mathrm{NCH}_{2} \mathrm{CH}_{2}, 2 \mathrm{H}$ ), 5.02 (br s, $\mathrm{CH}_{2} \mathrm{C}_{6} \mathrm{H}_{5}, 2 \mathrm{H}$ ), 5.15 (s, $\mathrm{C}_{5} \mathrm{H}_{5}, 5 \mathrm{H}$ ), 6.24 (s, $\left.\mathrm{CH}_{2} \mathrm{C}_{6} \mathrm{H}_{4} \mathrm{NO}_{2}, 2 \mathrm{H}\right), 6.76\left(\mathrm{~d},{ }^{3} J_{H H}=6 \mathrm{~Hz}, \mathrm{NCH}, 2 \mathrm{H}\right), 7.24-7.33\left(\mathrm{~m}, \mathrm{C}_{6} H_{5}, 5 \mathrm{H}\right), 7.43\left(\mathrm{~d},{ }^{3} J_{H H}\right.$ $\left.=7 \mathrm{~Hz}, \mathrm{C}_{6} H_{4}, 2 \mathrm{H}\right), 8.25\left(\mathrm{~d},{ }^{3} J_{H H}=8 \mathrm{~Hz}, \mathrm{C}_{6} H_{4}\right.$ adjacent to $\left.\mathrm{NO}_{2}, 2 \mathrm{H}\right) .{ }^{13} \mathrm{C}\left\{{ }^{1} \mathrm{H}\right\}-\mathrm{NMR}\left(\mathrm{CDCl}_{3}\right.$, $\left.\delta_{\mathrm{C}}\right) 37.2\left(\mathrm{~s}, \mathrm{CH}_{2} \mathrm{CH}_{2} \mathrm{C}_{6} \mathrm{H}_{5}\right), 53.7\left(\mathrm{~s}, \mathrm{CH}_{2} \mathrm{CH}_{2} \mathrm{C}_{6} \mathrm{H}_{5}\right), 55.3\left(\mathrm{~s}, \mathrm{CH}_{2} \mathrm{C}_{6} \mathrm{H}_{4} \mathrm{NO}_{2}\right), 92.0\left(\mathrm{~s}, C_{5} \mathrm{H}_{5}\right)$, $123.7(\mathrm{~s},\{\mathrm{PhEt}\} \mathrm{NCH}), 124.3\left(\mathrm{~s},\left\{4-\mathrm{NO}_{2} \mathrm{Bn}\right\} \mathrm{NCH}\right), 127.1\left(\mathrm{~s}, C_{6} \mathrm{H}_{5}\right), 128.5\left(\mathrm{~s}, C_{6} \mathrm{H}_{4}\right), 128.9$ (s, $\left.C_{6} \mathrm{H}_{5}\right), 129.1\left(\mathrm{~s}, C_{6} \mathrm{H}_{4}\right.$ adjacent to $\left.\mathrm{NO}_{2}\right), 129.6\left(\mathrm{~s}, C_{6} \mathrm{H}_{5}\right), 132.3\left(\mathrm{~s}, C_{6} \mathrm{H}_{5}\right), 133.2\left(\mathrm{~s}, C_{6} \mathrm{H}_{5}\right)$, 137.8 (s, ipso- $C_{6} \mathrm{H}_{5}$ of $\mathrm{PhEt}$ ), 143.9 (s, ipso- $C_{6} \mathrm{H}_{4}$ of 4- $\mathrm{NO}_{2} \mathrm{Bn}$ ), 147.9 (s, ipso- $C_{6} \mathrm{H}_{4}$ containing $\left.\mathrm{NO}_{2}\right), 164.8(\mathrm{~s}, \mathrm{NCN}) . \mathrm{CHN}(\%)$ : $\left[\mathrm{C}_{18} \mathrm{H}_{17} \mathrm{BrN}_{3} \mathrm{NiO}_{2}\right]: \mathrm{C}, 53.67(54.06), \mathrm{H}$, 4.09(4.34), N, 8.39(8.22). MS (ESI): m/z 509.26 (M+).

[CpNiBr\{Im(4-NO $\left.\left.\left.\mathrm{I}_{2} \mathrm{Bn}\right)_{2}\right\}\right]$ (9): Yield: 59\%. IR $\left(\mathrm{cm}^{-1}\right): 3098(v(=\mathrm{CH}), \mathrm{m}), 3071(v(=\mathrm{CH}), \mathrm{w})$, $2927(v(-\mathrm{CH}), \mathrm{m}), 2857(v(-\mathrm{CH}), \mathrm{w}), 1679$ (v(NO), asym, m), $1598(\mathrm{~s}), 1515(\delta(-\mathrm{CH})$, sym, s), $1444(\delta(-\mathrm{CH})$, sym, s), 1409 ( $\delta(-\mathrm{CH})$, asym, m), 1342 ( $\delta(-\mathrm{CH}), \mathrm{s}), 1237$ (v(-CN), s), 1179 (v(NO), sym, m), $1109(\mathrm{~m}), 1014(v(\mathrm{CN}), \mathrm{w}), 858(\mathrm{~m}), 798(\delta(=\mathrm{CH}), \mathrm{s}), 733(\delta(-\mathrm{CH}), \mathrm{s}) .{ }^{1} \mathrm{H}-$ $\operatorname{NMR}\left(\mathrm{CDCl}_{3}, \delta_{\mathrm{H}}\right) 5.28\left(\mathrm{~s}, \mathrm{C}_{5} \mathrm{H}_{5}, 5 \mathrm{H}\right), 6.18\left(\mathrm{dd},{ }^{2} J_{H H}=12\right.$ and $\left.52 \mathrm{~Hz}, \mathrm{CH}_{2} \mathrm{C}_{6} \mathrm{H}_{4} \mathrm{NO}_{2}, 4 \mathrm{H}\right)$, 6.42 (br s, $\mathrm{NCH}, 2 \mathrm{H}), 7.30-7.53\left(\mathrm{~m}, \mathrm{C}_{6} H_{4}, 4 \mathrm{H}\right), 8.13\left(\mathrm{~d},{ }^{3} J_{H H}=6 \mathrm{~Hz}, \mathrm{C}_{6} H_{4}\right.$ adjacent to $\mathrm{NO}_{2}$, $4 \mathrm{H}) .{ }^{13} \mathrm{C}\left\{{ }^{1} \mathrm{H}\right\}$-NMR $\left(\mathrm{CDCl}_{3}, \delta_{\mathrm{C}}\right) 41.5\left(\mathrm{~s}, \mathrm{CH}_{2}\right), 43.1\left(\mathrm{~s}, \mathrm{CH}_{2}\right), 92.1\left(\mathrm{~s}, C_{5} \mathrm{H}_{5}\right), 123.6(\mathrm{~s}, \mathrm{NCH})$, $123.7(\mathrm{~s}, \mathrm{NCH}), 128.6\left(\mathrm{~s}, C_{6} \mathrm{H}_{5}\right), 129.2\left(\mathrm{~s}, C_{6} \mathrm{H}_{5}\right), 144.0\left(\mathrm{~s}\right.$, ipso- $C_{6} \mathrm{H}_{4}$ of 4- $\left.\mathrm{NO}_{2} \mathrm{Bn}\right), 148.1$ (s, ipso- $C_{6} \mathrm{H}_{4}$ adjacent to $\mathrm{NO}_{2}$ ), 164.8 (s, $\mathrm{NCN}$ ). $\mathrm{CHN}(\%)$ : $\left[\mathrm{C}_{17} \mathrm{H}_{14} \mathrm{BrN}_{4} \mathrm{NiO}_{4}\right]$ : $\mathrm{C}, 48.73(48.75)$, H, 3.38(3.53), N, 10.52(10.34). MS (ESI): m/z 539.97 (M+).

\subsection{General procedure for the Suzuki-Miyaura coupling reactions}

To a round-bottom flask containing the substrate $\left(2.8 \times 10^{-4} \mathrm{~mol}\right), \mathrm{K}_{3} \mathrm{PO}_{4}\left(2.6\right.$ eq., $7.2 \times 10^{-4}$ $\mathrm{mol})$ and $\mathrm{PhB}(\mathrm{OH})_{2}\left(1.3 \mathrm{eq}, 3.6 \times 10^{-4} \mathrm{~mol}\right)$, was added $[\mathrm{CpNiBr}(\mathrm{NHC})]\left(3 \mathrm{~mol} \%, 8.3 \times 10^{-6}\right.$ mol) and toluene $(4 \mathrm{~mL})$ and the subsequent reaction mixture was stirred at $90^{\circ} \mathrm{C}$ for 60 
minutes. The mixture was left to cool, and passed through a pad of silica. $1 \mathrm{~mL}$ of the resulting light yellow solution was then analysed directly by GC/MS using hexadecane as internal standard upon which the coupling yields were based. All yields are based on the average of three runs.

\subsection{X-ray crystallography of compounds $\mathbf{L 3}, \mathbf{L 4}, \mathbf{1}, \mathbf{2}, \mathbf{3}$, and 4}

Single crystal diffraction studies of compounds $\mathbf{L 3}, \mathbf{L 4}, \mathbf{1}, \mathbf{2}, \mathbf{3}$, and 4 were done using Quazar multi-layer optics monochromated Mo Ka radiation $(\mathrm{k}=0.71069 \AA)$ ) on a Bruker D8 Venture kappa geometry diffractometer with duo I $\mu$ s sources, a Photon 100 CMOS detector and APEX II control software [29]. All X-ray diffraction measurements were performed at 150(2) K. Data reduction was performed using SAINT+ [29], and the intensities were corrected for absorption using SADABS [30]. All structures were solved by direct methods with SHELXS-97 [31] using the OLEX2 [32] interface. All $\mathrm{H}$ atoms were placed in geometrically idealized positions and constrained to ride on their parent atoms. For a table containing the data collection and refinement parameters, see Supplementary Information.

\section{Results and Discussion}

\subsection{Synthesis of cyclopentadienyl nickel(II) bromide NHC complexes}

Because of the continued interest in the synthesis and interesting application of the large range of $[\mathrm{CpNiX}(\mathrm{NHC})](\mathrm{X}=\mathrm{Cl}, \mathrm{I})$ complexes, the inherent need to expand on the few existing bromo-analogues existed. In order to address this, we have synthesised a range of flexible symmetric- and asymmetric NHC bromide ligands according to the reported methods $[2,13,21]$ (see supplementary information), in high yields (> 85\%). This synthetic route involves the initial $\mathrm{N}$-functionalisation of imidazole with $\mathrm{RBr}$ (alkyl-, benzyl-, or phenylethyl halide) in the presence of $\mathrm{KOH} / \mathrm{K}_{2} \mathrm{CO}_{3}$, which after purification is further reacted with $\mathrm{R}$ 'Br to yield the respective $N, N^{\prime}$-functionalised imidazolium bromide salts. Each of the resulting NHC ligands bears $N$-alkyl, $N$-benzyl or $-N$-phenylethylmoieties that provide bulk through phenyl groups, as well as flexibility through $\mathrm{sp}^{3}$ methylene carbon linkers (Figure 2). 


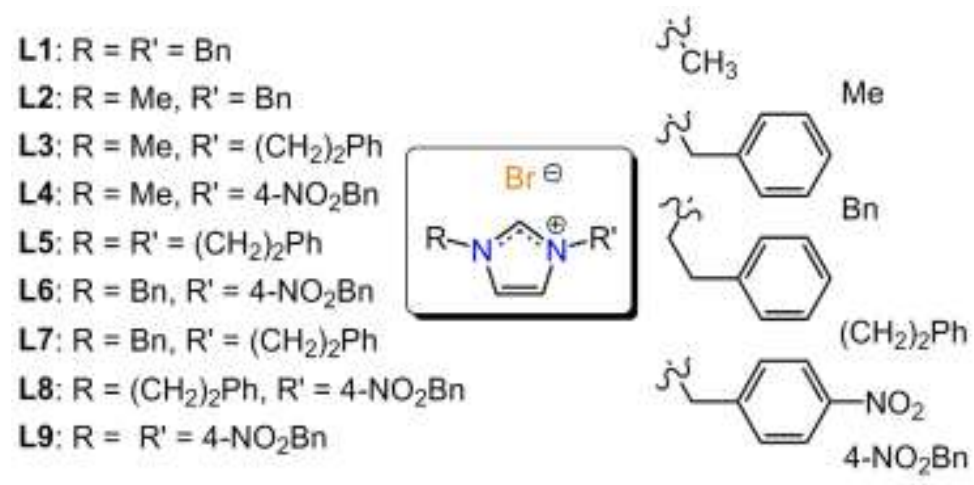

Figure 2: R and R' groups employed in ligands $\mathbf{L 1}-\mathbf{L 9}$.

Each of these $\mathrm{NHC}$ ligands was reacted with $\mathrm{NiCp}_{2}[12,14,19]$ to form the complexes [CpNiBr(NHC)] $(\mathbf{1}-\mathbf{9})$ with the loss of a cyclopentadiene ligand (Table 1). During these complexation reactions, a typical colour change from emerald green to blood red/red-brown was observed, with reaction times that varied between 3 and 6 hours. The more electron withdrawing NHC ligands of $\mathbf{4}, \mathbf{6}, \mathbf{8}$, and 9 proved to required longer reaction times, ranging from 6 - 18 hours to give the $\mathrm{Ni}(\mathrm{II})$ complexes in notably lower yields (59-74\%). Nolan et al. [12] have previously pointed out that longer reaction times were required whenever bulkier, as opposed to electron-withdrawing, NHC ligands were employed. The [CpNiBr(NHC)] complexes synthesised showed sufficient stability in deoxygenated commercial grade solvents of acetone, chloroform, and DCM.

In general the ${ }^{1} \mathrm{H}-\mathrm{NMR}$ spectra of complexes 1 - 9 correspond to those of similar complexes reported in literature $[11 \mathrm{~b}, 13,14,19,33]$. The cyclopentadienyl protons were observed as singlets between 5.12 and $5.28 \mathrm{ppm}$, along with the $\mathrm{NCH}$ protons appearing either as singlets, doublets, or quartets $\left({ }^{3} \mathrm{~J}_{\mathrm{HH}}=2-55 \mathrm{~Hz}\right)$ at $6.42-7.20 \mathrm{ppm}$, depending on the symmetrical nature of the NHC ligand employed. The ${ }^{13} \mathrm{C}-\mathrm{NMR}$ spectra of $\mathbf{1}-\mathbf{9}$ also conformed to literature, with the carbene carbon signal resonating between 161.3 (electron-donating NHCs) and 166.9 (electron-withdrawing NHCs) ppm. 
Table 1: Synthesis data of the $[\mathrm{CpNiBr}(\mathrm{NHC})]$ complexes $(\mathbf{1}-\mathbf{9})$

\begin{tabular}{clcc} 
& & & \\
Complex & {$[\mathrm{NHC}] \mathrm{Br}$} & Reaction & Yield (\%) \\
$\mathbf{1}$ & {$\left[\mathrm{HIm}(\mathrm{Bn})_{2}\right] \mathrm{Br}$} & time (h) & \\
$\mathbf{2}$ & {$[\mathrm{HIm}(\mathrm{Me})(\mathrm{Bn})] \mathrm{Br}$} & 3 & 77 \\
$\mathbf{3}$ & {$\left[\mathrm{HIm}(\mathrm{Me})\left(\left(\mathrm{CH}_{2}\right)_{2} \mathrm{Ph}\right)\right] \mathrm{Br}$} & 4 & 74 \\
$\mathbf{4}$ & {$\left[\mathrm{HIm}(\mathrm{Me})\left(4-\mathrm{NO}_{2} \mathrm{Bn}\right)\right] \mathrm{Br}$} & 6 & 69 \\
$\mathbf{5}$ & {$\left[\mathrm{HIm}\left((\mathrm{CH})_{2} \mathrm{Ph}\right)_{2}\right] \mathrm{Br}$} & 6 & 71 \\
$\mathbf{6}$ & {$\left[\mathrm{HIm}(\mathrm{Bn})\left(4-\mathrm{NO}_{2} \mathrm{Bn}\right)\right] \mathrm{Br}$} & 6 & 74 \\
$\mathbf{7}$ & {$\left[\mathrm{HIm}(\mathrm{Bn})\left(\left(\mathrm{CH}_{2}\right)_{2} \mathrm{Ph}\right)\right] \mathrm{Br}$} & 6 & 73 \\
$\mathbf{8}$ & {$\left[\mathrm{HIm}\left((\mathrm{CH})_{2} \mathrm{Ph}\right)(4-\right.$} & 7 & 70 \\
$\mathbf{9}$ & $\left.\left.\mathrm{NO}_{2} \mathrm{Bn}\right)\right] \mathrm{Br}$ & 16 & 59 \\
\hline
\end{tabular}

\subsection{X-ray crystallographic studies}

The molecular structures of complexes $\left[\mathrm{CpNiBr}\left\{\operatorname{Im}(\mathrm{Bn})_{2}\right\}\right](\mathbf{1}),[\mathrm{CpNiBr}\{\operatorname{Im}(\mathrm{Me})(\mathrm{Bn})\}](\mathbf{2})$, $\left[\mathrm{CpNiBr}\left\{\operatorname{Im}(\mathrm{Me})\left(\left(\mathrm{CH}_{2}\right)_{2} \mathrm{Ph}\right)\right\}\right]$ (3), and $\left[\mathrm{CpNiBr}\left\{\operatorname{Im}(\mathrm{Me})\left(4-\mathrm{NO}_{2} \mathrm{Bn}\right)\right\}\right]$ (4) have been confirmed by single crystal X-ray diffraction, and are included in Figures 3 and 4 along with the structures of $\mathbf{L 3}$ and $\mathbf{L 4}$. All crystallographic data and data collection parameters are included in the supplementary information. With the cyclopentadienyl ligand considered as a single ligand, complexes $\mathbf{1}-\mathbf{4}$ may be considered to exhibit a distorted trigonal planar geometry around the central metal atom, with $\mathrm{Cp}_{\text {cent }}-\mathrm{Ni1}-\mathrm{Br} 1 \approx \mathrm{Cp}_{\text {cent }}-\mathrm{Ni}-\mathrm{C} 1 \approx 133^{\circ}$. In addition, the angle between the mean imidazolyl (NHC) plane and Ni1-Br1 bond ranges between $92.865^{\circ}-94.519^{\circ}$ for $\mathbf{1}, \mathbf{3}$ and 4 . This angle, however, increases to $96.687^{\circ}$ for $\mathbf{2}$, which contains the least bulky NHC ligand of the four complexes. This is the result of the Cp and $\mathrm{Br}$ ligands being more conveniently arranged around Ni1 with the smaller NHC ligand. 


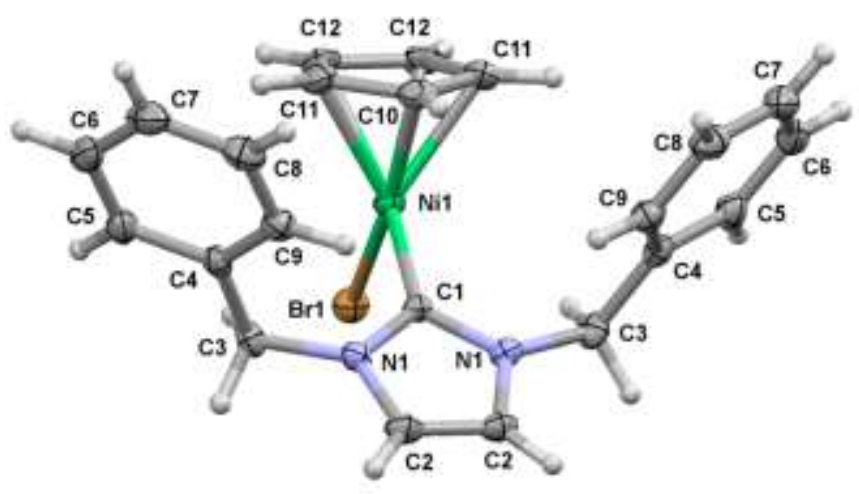

1

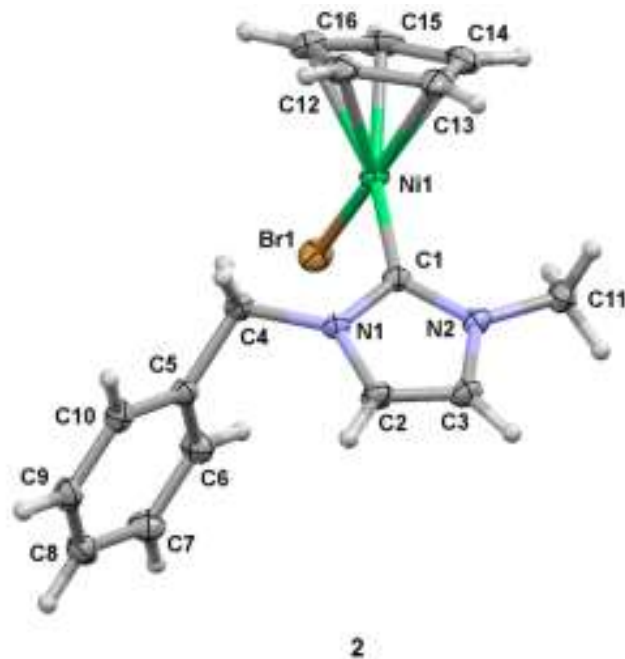

2

Figure 3: Perspective views of $\left[\mathrm{CpNiBr}\left\{\operatorname{Im}(\mathrm{Bn})_{2}\right\}\right](\mathbf{1})$ and $[\mathrm{CpNiBr}\{\operatorname{Im}(\mathrm{Me})(\mathrm{Bn})\}]($ 2) with thermal ellipsoids drawn at $50 \%$ probability level.

The key descriptors of both structures compare well with similar [CpNiBr(NHC)] complexes

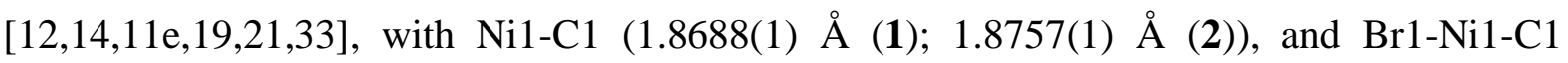
$\left(94.107(1)^{\circ}(\mathbf{1}) ; 96.201(3)^{\circ}(\mathbf{2})\right)$ (Table 2). The molecular structures obtained for ligands $\left[\operatorname{Im}(\mathrm{Me})\left(\left(\mathrm{CH}_{2}\right)_{2} \mathrm{Ph}\right)\right] \mathrm{Br}(\mathbf{L 3})$ and $\left[\operatorname{Im}(\mathrm{Me})\left(4-\mathrm{NO}_{2} \mathrm{Bn}\right)\right] \mathrm{Br}(\mathbf{L 4})$, and their corresponding $\mathrm{Ni}(\mathrm{II})-$ complexes (3 and 4 respectively) are included in Figure 4. The Br1-Ni1-C1 angles of $93.965(3)^{\circ}$ (3) and $94.227(6)^{\circ}$ (avg.) (4) correspond well with those of $\mathbf{1}$ and $\mathbf{2}$, and fall within the range $92.9(2)^{\circ}-98.41(9)^{\circ}$ reported for most of the $[\mathrm{CpNiX}(\mathrm{NHC})](\mathrm{X}=\mathrm{Cl}, \mathrm{Br}, \mathrm{I})$ analogues [12,14,11e,19,21,33]. The flexibility in the range of NHC ligands employed, before and after coordination is structurally demonstrated through the $\mathrm{N} 1-\mathrm{C} 1-\mathrm{X}$ and $\mathrm{N} 2-\mathrm{C} 1-\mathrm{X}$ bond angles, as well as the $\mathrm{C}-\mathrm{N} 1-\mathrm{C} 1-\mathrm{X}$ and $\mathrm{C}-\mathrm{N} 2-\mathrm{C} 1-\mathrm{X}$ torsion angles, where $\mathrm{X}=\mathrm{H}, \mathrm{Ni}$. In sterically demanding environments the $N$-substituents tend to "fold-out", such that the bulky moieties are directed away from the metal centre, which is observed through the C-N-C and C-N-C-Ni angles. The N1-C1-X angles $\left(123.969(1)^{\circ}(\mathbf{L 3}) ; 125.752(4)^{\circ}(\mathbf{L 4})\right)$, as well as the N2-C1-X angles $\left(127.823(2)^{\circ}(\mathbf{L 3}) ; 125.753(2)^{\circ}(\mathbf{L 4})\right)$ in general increased after complexation to $127.623(4)^{\circ}$ (3) and $127.469(9)^{\circ}$ (avg.) (4), and $128.052(4)^{\circ}$ (3) and $127.950(9)^{\circ}$ (avg.) (4), respectively. This shows the compromise the benzyl $(\mathbf{1}, \mathbf{2})$, phenylethyl (3) and 4- $\mathrm{NO}_{2}$-benzyl (4) groups make to fit around an already crowded nickel centre bearing cyclopentadienyl and bromide ligands. The C-N1-C1-X and C-N2-C1-X (X = $\mathrm{H}, \mathrm{Ni}$ ) torsion angles indicated planarity that can be associated with delocalization of electron density from the ring system to the $X$ atom $\left(4.226(3)^{\circ}\right.$ and $-5.210(3)^{\circ}(\mathbf{L 3}) ; 2.132(7)^{\circ}$ and $1.030(7)^{\circ}(\mathbf{L 4}) ; 1.020(9)^{\circ}$ and $1.577(9)^{\circ}(3) ; 2.313(2)^{\circ}\left(\right.$ avg.) and $1.961(2)^{\circ}$ (avg.) (4)). No 


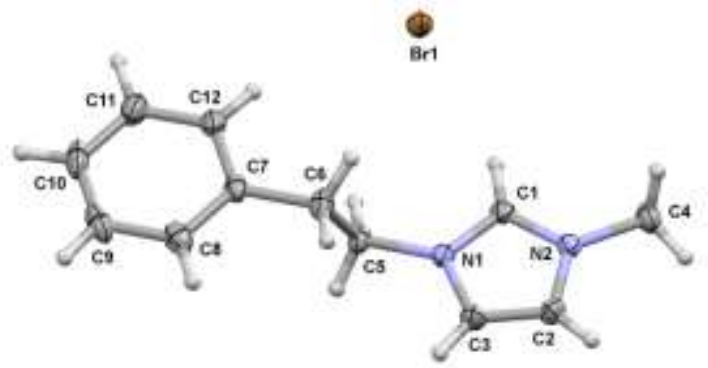

L3

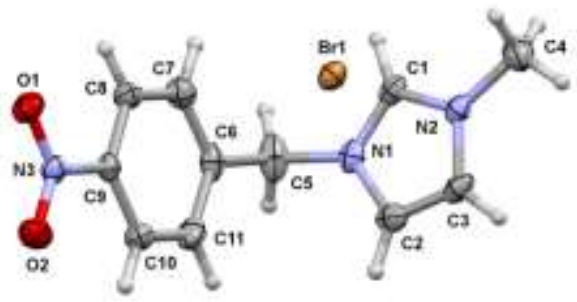

L4
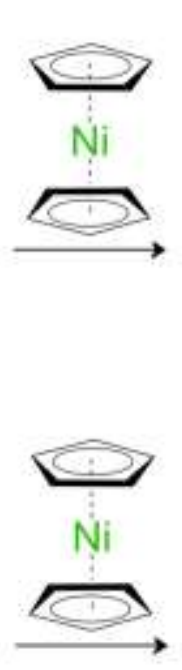

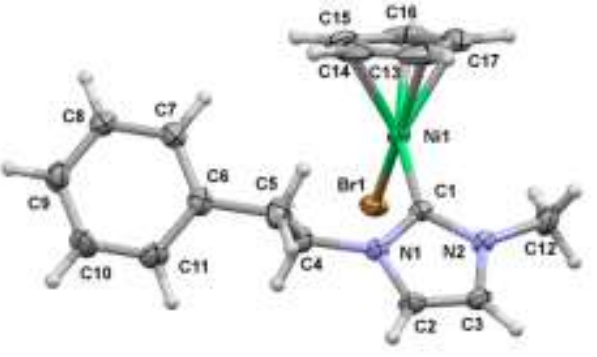

3

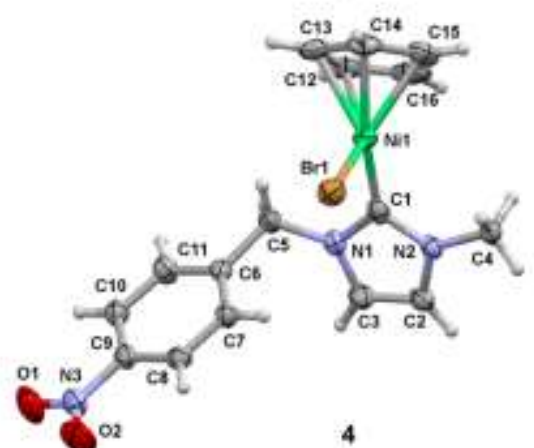

Figure 4: Perspective views of $\left[\mathrm{HIm}(\mathrm{Me})\left(\left(\mathrm{CH}_{2}\right)_{2} \mathrm{Ph}\right)\right] \mathrm{Br}(\mathbf{L 3}),\left[\mathrm{HIm}(\mathrm{Me})\left(4-\mathrm{NO}_{2} \mathrm{Bn}\right)\right] \mathrm{Br}(\mathbf{L 4})$, along with their corresponding $[\mathrm{CpNiBr}(\mathrm{NHC})]$ complexes $(\mathbf{3} ; \mathbf{4})$ with thermal ellipsoids drawn at $50 \%$ probability level. Half a molecule of $\mathrm{C}_{6} \mathrm{H}_{6}$ (solvent of crystallization) in $\mathbf{3}$ has been omitted for clarity.

Table 2: Selected bond distances $(\AA)$ and angles $\left({ }^{\circ}\right)$ for compounds $\mathbf{L 3}, \mathbf{L 4}, \mathbf{1}, \mathbf{2}, \mathbf{3}$ and 4

\begin{tabular}{|c|c|c|c|c|c|c|}
\hline Description & L3 & L4 & 1 & 2 & $3^{b}$ & $4^{\mathrm{c}}$ \\
\hline Ni-C1 & -- & -- & $1.8688(1)$ & $1.8757(1)$ & $1.8753(1)$ & $\begin{array}{l}1.8557(1) \\
1.8806(2)\end{array}$ \\
\hline Ni1-Br1 & -- & -- & $2.3427(1)$ & $2.3253(1)$ & $2.3272(1)$ & $\begin{array}{l}2.3166(2) \\
2.3154(2)\end{array}$ \\
\hline Br1-Ni1-C1 & -- & -- & $94.107(1)$ & $96.201(3)$ & $93.965(3)$ & $\begin{array}{l}94.528(6) \\
93.925(6)\end{array}$ \\
\hline N1-C1-X (X = Ni, H) & $123.969(1)$ & $125.752(4)$ & $127.706(2)$ & $127.189(4)$ & $127.623(4)$ & $\begin{array}{l}127.279(8) \\
127.658(9)\end{array}$ \\
\hline N2-C1- X (X = Ni, H) & $127.823(2)$ & $125.753(2)$ & $127.706(2)$ & $128.375(4)$ & $128.052(4)$ & $\begin{array}{l}129.040(9) \\
126.860(8)\end{array}$ \\
\hline $\mathrm{C}^{\mathrm{a}}-\mathrm{N} 1-\mathrm{C} 1$ & $125.375(1)$ & $125.600(3)$ & $123.826(2)$ & $124.104(3)$ & $124.671(6)$ & $\begin{array}{l}123.199(9) \\
124.046(1)\end{array}$ \\
\hline $\mathrm{C}^{\mathrm{a}}-\mathrm{N} 2-\mathrm{C} 1$ & $125.622(1)$ & $125.994(2)$ & $123.826(2)$ & $124.661(3)$ & $124.123(6)$ & $\begin{array}{l}122.975(1) \\
124.358(1)\end{array}$ \\
\hline$C^{\mathrm{a}}-\mathrm{N} 1-\mathrm{C} 1-\mathrm{X}(\mathrm{X}=\mathrm{Ni}, \mathrm{H})$ & $4.226(3)$ & $2.132(7)$ & $6.267(3)$ & $3.587(7)$ & $1.020(9)$ & $\begin{array}{l}2.343(2) \\
2.282(2)\end{array}$ \\
\hline$C^{\mathrm{a}}-\mathrm{N} 2-\mathrm{C} 1-\mathrm{X}(\mathrm{X}=\mathrm{Ni}, \mathrm{H})$ & $-5.210(3)$ & $-1.030(7)$ & $-6.267(3)$ & $-4.400(7)$ & $1.577(9)$ & $\begin{array}{c}0.053(2) \\
-3.868(2)\end{array}$ \\
\hline
\end{tabular}


mentionable changes in the C-N1-C1 and C-N2-C1 bond angles were observed either before or after complexation, however. Collectively, the ability of the ligand salts (L3, L4) to compensate for a decrease of spatial freedom when coordinated $(\mathbf{3}, \mathbf{4})$, is shown through the "folding-out" and flexibility of the $N$-substituted groups. This functionality of the ligands and corresponding complexes provide a unique way of introducing the required steric bulk and flexibility [12] in the ligand systems of Suzuki-Miyaura active catalyst complexes.

\subsection{Suzuki-Miyaura coupling activity}

The Suzuki-Miyaura reaction is usually carried out with 4-halo acetophenone and phenylboronic acid as coupling partners to yield 4-acetylbiphenyl (major, $70-100 \%$ conversion) and very low yields of the homo-coupled by-product, 4,4'-diacetylbiphenyl (minor, $0-16 \%$ ) in less than 60 minutes. Complexes $1-9$ were employed as catalysts in the Suzuki-Miyaura (SM) coupling reaction of the aryl chloride, 4-chlorobenzaldehyde and $\mathrm{PhB}(\mathrm{OH})_{2}$. This is the first instance where the more challenging [14,20b,34] chloro-aryl carboxaldehydes are employed as substrates using $\mathrm{CpNiBr}(\mathrm{NHC})$ complexes, as opposed to the usual chloro- and bromo aryl ketones with $[\mathrm{CpNiX}(\mathrm{NHC})](\mathrm{X}=\mathrm{Cl}, \mathrm{I})$ complexes as catalysts [14,19,20c,33]. All complexes $1-9$ exhibited catalytic activity under optimized reaction conditions, i.e. stirred suspensions in toluene with 2.6 eq. $\mathrm{K}_{3} \mathrm{PO}_{4}, 1.3$ eq. $\mathrm{PhB}(\mathrm{OH})_{2}$, and $3 \mathrm{~mol} \%$ catalyst at $90^{\circ} \mathrm{C}$, to give 4-carboxaldehydebiphenyl (CBP) (53 - 79\% conversion) as the major product within one hour (Table 3). Surprisingly, formation of minute amounts of the homo-coupled product, 4,4'-dicarboxaldehydebiphenyl, and a significant amount of biphenyl as by-products $(15-30 \%)$ were observed. This result was found to be directly related to the $\mathrm{N}$-substituted groups of the different $\mathrm{Ni}$ (II)-NHC complexes involved in each isolated reaction, where the more electron-withdrawing benzyl groups tend to yield slightly higher yields of biphenyl, with consequential lower yields of CBP. 
Table 3: Suzuki-Miyaura reaction of 4-chlorobenzaldehyde with phenylboronic acid catalysed by [CpNiBr(NHC) complexes $(\mathbf{1}-\mathbf{9})$

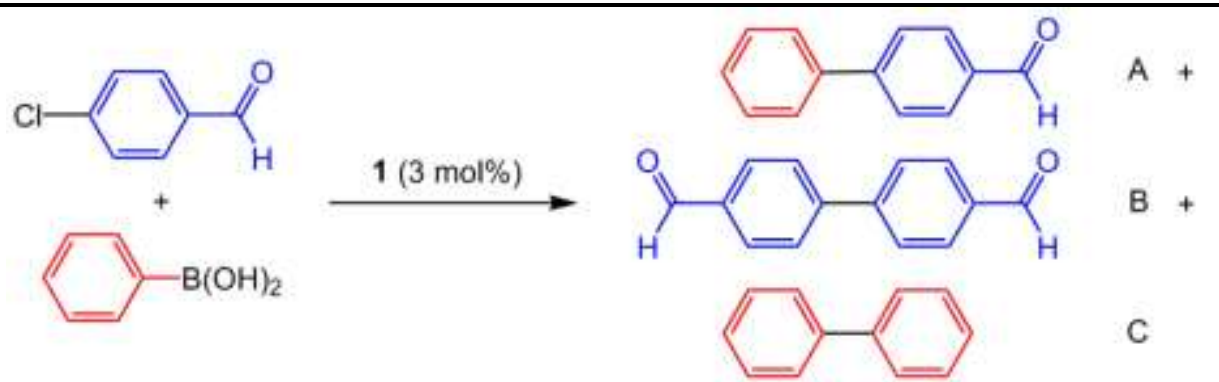

\begin{tabular}{ccccccccc}
\hline Entry & $\begin{array}{c}\text { Cat. } \\
(\mathbf{m o l} \%)\end{array}$ & Solvent & $\mathbf{B a s e}$ & $\begin{array}{c}\text { Time } \\
(\mathbf{m i n})\end{array}$ & $\begin{array}{c}\text { Temp } \\
\left({ }^{\circ} \mathbf{C}\right)\end{array}$ & $\mathbf{S : P B A : B ^ { \mathbf { a } }}$ & Conv. $(\boldsymbol{\%})^{\mathbf{b}}$ & A:B:C \\
\hline $\mathbf{1}$ & $\mathbf{1}(3)$ & toluene & $\mathrm{K}_{3} \mathrm{PO}_{4}$ & 60 & 90 & $1: 1.3: 2.6$ & 79 & $79: 0: 21$ \\
$\mathbf{2}$ & $\mathbf{1}(3)$ & toluene & $\mathrm{K}_{2} \mathrm{HPO}_{4}$ & 60 & 90 & $1: 1.3: 2.6$ & 33 & $76: 0: 24$ \\
$\mathbf{3}$ & $\mathbf{1}(3)$ & toluene & $\mathrm{K}_{2} \mathrm{CO}_{3}$ & 60 & 90 & $1: 1.3: 2.6$ & 62 & $75: 0: 25$ \\
$\mathbf{4}$ & $\mathbf{1}(3)$ & toluene & $\mathrm{NEt}_{3}$ & 60 & 90 & $1: 1.3: 2.6$ & 4 & $24: 3: 73$ \\
$\mathbf{5}$ & $\mathbf{1}(3)$ & toluene & $\mathrm{K}_{3} \mathrm{PO}_{4}$ & 30 & 90 & $1: 1.3: 2.6$ & 43 & $73: 0: 27$ \\
$\mathbf{6}$ & $\mathbf{1}(3)$ & toluene & $\mathrm{K}_{3} \mathrm{PO}_{4}$ & 120 & 90 & $1: 1.3: 2.6$ & 87 & $69: 0: 31$ \\
$\mathbf{7}$ & $\mathbf{1}(3)$ & toluene & $\mathrm{K}_{3} \mathrm{PO}_{4}$ & 60 & 60 & $1: 1.3: 2.6$ & 46 & $65: 0: 35$ \\
$\mathbf{8}$ & $\mathbf{1}(3)$ & toluene & $\mathrm{K}_{3} \mathrm{PO}_{4}$ & 60 & 25 & $1: 1.3: 2.6$ & 24 & $58: 0: 42$ \\
$\mathbf{9}$ & $\mathbf{1}(3)$ & DMP & $\mathrm{K}_{3} \mathrm{PO}_{4}$ & 60 & 90 & $1: 1.3: 2.6$ & 42 & $65: 0: 35$ \\
$\mathbf{1 0}$ & $\mathbf{1}(3)$ & CH ${ }_{3} \mathrm{CN}$ & $\mathrm{K}_{3} \mathrm{PO}_{4}$ & 60 & 90 & $1: 1.3: 2.6$ & 23 & $43: 0: 57$ \\
$\mathbf{1 1}$ & $\mathbf{1}(0)$ & toluene & $\mathrm{K}_{3} \mathrm{PO}_{4}$ & 60 & 90 & $1: 1.3: 2.6$ & 5 & $9: 0: 91$ \\
$\mathbf{1 2}$ & $\mathbf{1}(3)$ & toluene & $\mathrm{K}_{3} \mathrm{PO}_{4}$ & 60 & 90 & $1: 1.1: 2.2$ & 59 & $81: 0: 19$ \\
$\mathbf{1 3}$ & $\mathbf{2}(3)$ & toluene & $\mathrm{K}_{3} \mathrm{PO}_{4}$ & 60 & 90 & $1: 1.3: 2.6$ & 73 & $79: 0: 21$ \\
$\mathbf{1 4}$ & $\mathbf{3}(3)$ & toluene & $\mathrm{K}_{3} \mathrm{PO}_{4}$ & 60 & 90 & $1: 1.3: 2.6$ & 69 & $70: 0: 30$ \\
$\mathbf{1 5}$ & $\mathbf{4}(3)$ & toluene & $\mathrm{K}_{3} \mathrm{PO}_{4}$ & 60 & 90 & $1: 1.3: 2.6$ & 71 & $83: 1: 16$ \\
$\mathbf{1 6}$ & $\mathbf{5}(3)$ & toluene & $\mathrm{K}_{3} \mathrm{PO}_{4}$ & 60 & 90 & $1: 1.3: 2.6$ & 76 & $78: 0: 22$ \\
$\mathbf{1 7}$ & $\mathbf{6 ( 3 )}$ & toluene & $\mathrm{K}_{3} \mathrm{PO}_{4}$ & 60 & 90 & $1: 1.3: 2.6$ & 62 & $78: 0: 22$ \\
$\mathbf{1 8}$ & $\mathbf{7}(3)$ & toluene & $\mathrm{K}_{3} \mathrm{PO}_{4}$ & 60 & 90 & $1: 1.3: 2.6$ & 73 & $78: 0: 22$ \\
$\mathbf{1 9}$ & $\mathbf{8}(3)$ & toluene & $\mathrm{K}_{3} \mathrm{PO}_{4}$ & 60 & 90 & $1: 1.3: 2.6$ & 57 & $66: 0: 34$ \\
$\mathbf{2 0}$ & $\mathbf{9}(3)$ & toluene & $\mathrm{K}_{3} \mathrm{PO}_{4}$ & 60 & 90 & $1: 1.3: 2.6$ & 53 & $77: 0: 23$ \\
\hline
\end{tabular}

General reaction conditions: 4-chlorobenzaldehyde $(0.28 \mathrm{mmol})$, phenylboronic acid (0.36 mmol), base $(0.72$

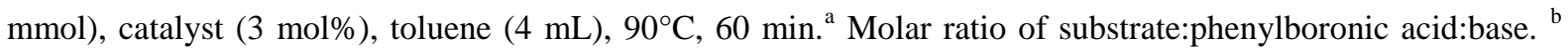
Conversion based on internal standard, average value of three runs.

In the optimization process of the SM reaction we found, as with other groups [14,19,33], that $\mathrm{K}_{3} \mathrm{PO}_{4}$ as base is by far more effective compared to $\mathrm{K}_{2} \mathrm{HPO}_{4}, \mathrm{~K}_{2} \mathrm{CO}_{3}$ or $\mathrm{NEt}_{3}$ (entries 14). An increase in reaction time, or with increased temperature, led to increased yields 
(entries 5-6). We focused on a direct comparison of catalytic efficiencies with those reported and therefore employed similar reaction conditions $\left(90^{\circ} \mathrm{C}, 60 \mathrm{~min}\right)$, for which we obtained moderate to high conversions. An immediate lowering in conversion was observed upon lowering of the reaction temperature (entries 7-8). A reaction time of 60 minutes is still considered fast for Ni-catalysed SM reactions [19], especially without added additives such as $\mathrm{PPh}_{3}$ [19], whereas product formation occurred within the first 5 minutes. Polar aprotic $\mathrm{CH}_{3} \mathrm{CN}$ and dimethoxypropane proved inefficient (entries 9-10), whereas toluene proved superior as the solvent of choice for aryl coupling reactions that can withstand the temperatures involved. Using toluene as solvent, a stabilisation effect might also be significant, especially if air- and moisture sensitive Ni-NHC catalytic species are involved, through soft $\pi$-interactions of the $N$-phenyl-containing groups of the NHC ligands. This effect is further illustrated when lower catalyst concentrations are used providing lower CBP conversions (entry 11), and might indicate fast catalyst deactivation [19]. In an attempt to decrease the biphenyl product formation, separate decreases in $\mathrm{K}_{3} \mathrm{PO}_{4}$ (2.2 eq.) and $\mathrm{PhB}(\mathrm{OH})_{2}$ amounts led to an immediate decrease of $\mathrm{CBP}$ yield, with no significant proportional decrease of biphenyl formation (entry 12).

A significant variation of CBP yields was observed among each of the complexes 1 - 9 . Under the optimized conditions, $\left[\mathrm{CpNiBr}\left\{\operatorname{Im}(\mathrm{Bn})_{2}\right\}\right]$ (1) performed best, with $79 \%$ conversion in 60 minutes. An initial turn-over frequency (TOF) of $320 \mathrm{~h}^{-1}$ was observed for 1. This is much higher than the reported $190 \mathrm{~h}^{-1}\left(\left[\mathrm{Cp} * \mathrm{Ni}(\mathrm{NCMe})\left\{\operatorname{Im}\left(\mathrm{iPr}_{2} \mathrm{Ph}\right)_{2}\right\}\right] \mathrm{PF}_{6}\right)$ [33], and comparable to $352 \mathrm{~h}^{-1}$ ([CpNiI $\left.\left.\{\operatorname{Im}(\mathrm{Mes})(\mathrm{nBu})\}\right]\right)$, observed to have the best TOF for a $\mathrm{Ni}$ (II)-catalysed SM coupling in the absence of reductant and/or co-catalysts [19]. Amongst complexes 1 -9, a general trend was observed where the more electron-withdrawing NHCbearing complexes led to the lowest $\mathrm{CBP}$ yields, i.e. $\left[\mathrm{CpNiBr}\left\{\operatorname{Im}\left(4-\mathrm{NO}_{2} \mathrm{Bn}\right)_{2}\right\}\right](9)$ produced only 53\% conversion (entry 20). This could be explained by the relative nickel complex stabilities once dehalogenation and/or reduction by $\mathrm{K}_{3} \mathrm{PO}_{4}$ have taken place. The more efficient electron-donating ancillary ligands stabilise the sensitive intermediates, and ultimately depress catalyst decomposition [20b]. This effect was also observed by the groups of Ritleng [19] and Buchowicz [14], where they found that electron-donating, bulky $N$ substituents lead to higher 4-acetylbiphenyl conversions, and the minimization of the homocoupled product formation. Moreover, Nolan et al. [12] found that the use of bulky NHC ligands stabilises the intermediate nickel species, while flexible NHC ligands proved even more useful to allow for unhindered substrate coordination. The latter observation 
agrees with our findings, where accessibility to incoming substrates, and the reductive elimination step in the catalytic cycle is better facilitated with bulky, flexible benzyl- and phenethyl-bearing NHCs. The $\mathrm{sp}^{3}$-methylene carbon-linked NHCs allow for free rotation of the bulky phenyl groups, which could at any stage of the catalytic process find itself in close proximity to the metal centre through ligand/substrate interactions. This creates intra-ligand strain, which could increase the rate of reductive elimination in order to relieve steric strain before the following substrate coordinates.

In addition, we also investigated the effect of employing the non-activated bromobenzene, as well as activated electron-donating substrates in these coupling reactions, and found that the results conform to those reported $[6 b, 19,33]$ in that all coupling yields decreased dramatically (Table 4). Little conversion was observed for bromobenzene, and only $24 \%$ conversion for 2 bromopyridine. The electron-withdrawing 4-bromophenacylbromide showed most promising with a conversion of $37 \%$ and little or no signs of side-reactions, even with an acetyl bromide functionality present.

Table 4: Suzuki-Miyaura reaction of other substrates with phenylboronic acid catalysed by complex $\mathbf{1}^{\mathrm{a}, \mathrm{b}}$

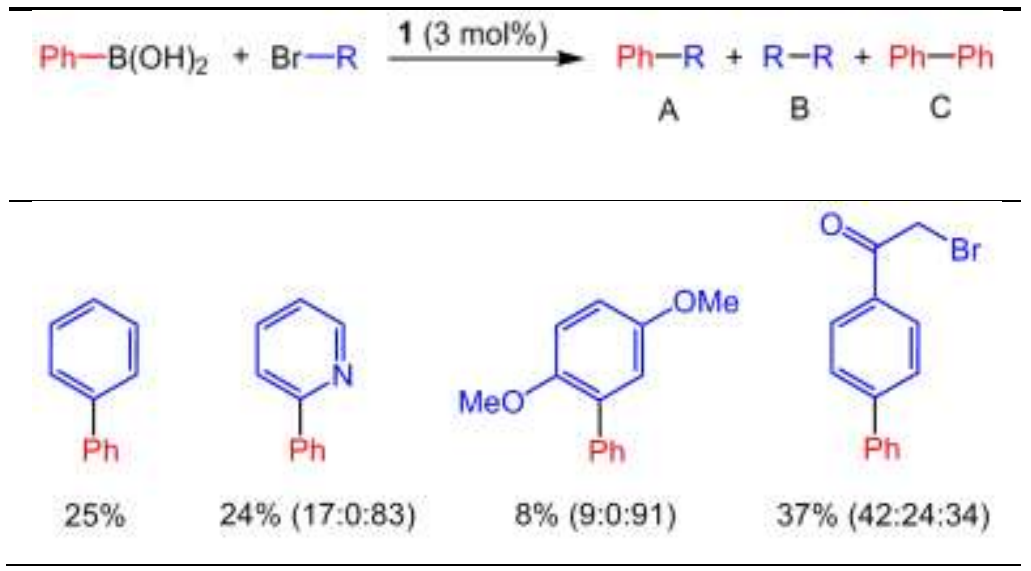

Reaction conditions: substrate $(0.28 \mathrm{mmol})$, phenylboronic acid $(0.36 \mathrm{mmol})$, base $(0.72 \mathrm{mmol}), 1$ (3 mol\%), toluene $(4 \mathrm{~mL}), 90^{\circ} \mathrm{C}, 60 \mathrm{~min} .{ }^{\mathrm{a}} \mathrm{GC}$ yields based on internal standard, average value of three runs. ${ }^{\mathrm{b}}$ Molar ratio of products $\mathrm{A}: \mathrm{B}: \mathrm{C}$, shown in parentheses.

According to the five important points required, as set out by Matsubara et al. [24] when designing a multi-functional nickel catalyst, the series [CpNiBr(NHC)] $(\mathbf{1}-\mathbf{9})$ conforms to the requirements. This is because complexes $\mathbf{1}-\mathbf{9}$ (i) are prepared in a facile fashion, (ii) are 
thermally and relatively air and moisture stable, (iii) easily form a coordinatively unsaturated site with mild bases, (iv) possess electron-donating ligands to stabilise the unsaturated site which accelerates the oxidative addition process, and (v) exhibit a range of steric substituents on the NHC ligand to enhance the reductive elimination reaction $[12,25]$.

\section{Conclusion}

A new range of flexible, electron-withdrawing and -donating NHC ligands, as well as their relatively air and moisture stable $[\mathrm{CpNiBr}(\mathrm{NHC})]$ complexes were synthesised directly from nickelocene, and characterised. Molecular structures of two of these [NHC]Br ligand salts, and four of the corresponding [ $\mathrm{CpNiBr}(\mathrm{NHC})]$ complexes were obtained and elucidated. This series of $\mathrm{Ni}(\mathrm{II})-\mathrm{NHC}$ complexes serves as an interesting expansion on the current limited series of bromo-analogues when compared with the vast series of $[\mathrm{CpNiX}(\mathrm{NHC})](\mathrm{X}=\mathrm{Cl}, \mathrm{I})$ complexes reported to date. The complexes $\mathbf{1}-\mathbf{9}$ efficiently catalysed the SM reaction of, amongst others, 4-chlorobenzaldehyde, which under optimized conditions gave 4carboxaldehydebiphenyl (major) and biphenyl (minor) in an average ratio of 80:20 respectively. The flexibility and bulkiness of the $N$-substituted groups on the NHC ligands provided accessibility to incoming substrates, while also facilitating the reductive elimination of the organic products. Among complexes 1 - 9, the more electron-donating NHC-bearing complexes were more efficient at the coupling of activated aryl halides and phenylboronic acid in the SM reactions investigated, as opposed to the electron-withdrawing NHC-bearing complexes.

\section{Supplementary material}

CCDC 1445421-1445426 contain the supplementary crystallographic data for compounds L3, L4, 1, 2, 3, and 4 respectively. Crystallographic files can be obtained from The Cambridge Crystallographic Data Centre via www.ccdc.cam.ac.uk/data_request/cif.

\section{Acknowledgements}

The National Research Foundation of South Africa (NRF) and the University of Pretoria (UP) are gratefully acknowledged for financial support (ML, FPM). Single crystal X-ray diffraction collections by Mr D. Liles are gratefully acknowledged as well as assistance with GC-MS studies by Dr Y. Naudé. 


\section{References}

[1] S. Gaillard, J-L. Renaud, Dalton Trans. 42 (2013) 7255-7270.

[2] O. Kühl, Chem. Soc. Rev. 36 (2007) 592-607.

[3] (a) B.K. Shaw, B.O. Patrick, M.D. Fryzuk, Organometallics. 31 (2012) 783-786. (b)

L. Mercs, G. Labat, A. Neels, A. Ehlers, M. Albrecht, Organometallics. 25 (2006) $5648-5656$.

[4] (a) T. Steinke, B.K. Shaw, H. Jong, B.O. Patrick, M.D. Fryzuk, Organometallics. 28 (2009) 2830-2836. (b) W.A. Herrmann, Angew. Chem. Int. Ed. 41 (2002) 1290-1309. (c) K.M. Hindi, M.J. Panzner, C.A. Tessier, C.L. Cannon, W.J. Youngs, Chem. Rev. 109 (2009) 3859-3884. (d) A. Kumar, P. Ghosh, Eur. J. Inorg. Chem. (2012) 39553969. (e) A. Gautier, F. Cisnetti, Metallomics. 4 (2012) 23-32.

[5] (a) F.E. Hahn, M.C. Jahnke, Angew. Chem. Int. Ed. 47 (2008) 3122-3172. (b) O. Schuster, L. Yang, H.G. Raubenheimer, M. Albrecht, Chem. Rev. 109 (2009) 34453478.

[6] (a) R.H. Crabtree, Coord. Chem. Rev. 257 (2013) 755-766. (b) C.-C. Ho, S. Chatterjee, T-L. Wu, K-T. Chan, Y-W. Chang, T-H. Hsiao, H.M. Lee, Organometallics. 28 (2009) 2837-2847.

[7] O.R. Luca, B.A. Thompson, M.K. Takase, R.H. Crabtree, J. Organomet. Chem. 730 (2013) 79-83.

[8] (a) A.R. Naziruddin, A. Hepp, T. Pape, F.E. Hahn, Organometallics. 30 (2011) 58595866. (b) J. Li, J. Peng, Y. Bai, G. Lai, X. Li, J. Organomet. Chem. 696 (2011) 21162121. (c) J.M. Praetorius, C.M. Crudden, Dalton Trans. (2008) 4079-4094.

[9] (a) V. Miranda-Soto, D.B. Grotjahn, A.L. Cooksy, J.A. Golen, C.E. Moore, A.L. Rheingold, Angew. Chem. Int. Ed. 50 (2011) 631-635. (b) W.N.O. Wylie, A.J. Lough, R.H. Morris, Chem. Comm. 46 (2010) 8240-8242. (c) C. Pranckevicius, D.W. Stephan, Chem. Eur. J. 20 (2014) 6597-6602. (d) E. Becker, V. Stingl, G. Dazinger, M. Puchberger, K. Mereiter, K. Kirchner, J. Am. Chem. Soc. 128 (2006) 6572-6573. (e) D. Jantke, M. Cokoja, A. Pöthig, W.A. Herrmann, F.E. Kühn, Organometallics. 32 (2013) 741-744. (f) J. Witt, A. Pöthig, F.E. Kühn, W. Baratta, Organometallics. 32 (2013) 4042-4045.

[10] A.P. Prakasham, P. Ghosh, Inorg. Chim. Acta. 431 (2015) 61-100.

[11] (a) W. Buchowicz, A. Kozioł, L.B. Jerzykiewicz, T. Lis, S. Pasynkiewicz, A. Pęcherzewska, A. Pietrzykowski, J. Mol. Cat. A. 257 (2006) 118-123. (b) V. Ritleng, 
C. Barth, E. Brenner, S. Milosevic, M.J. Chetcuti, Organometallics. 27 (2008) 42234228. (c) R.A. Kelly III, N.M. Scott, S. Díez-González, E.D. Stevens, S.P. Nolan, Organometallics. 24 (2005) 3442-3447. (d) M. Henrion, M.J. Chetcuti, V. Ritleng, Chem. Comm. 50 (2014) 4624-4627. (e) A.M. Oertel, V. Ritleng, A. Busiah, A., L.F. Veiros, M.J. Chetcuti, Organometallics. 30 (2011) 6495-6498.

[12] A.R. Martin, Y. Makida, S. Meiries, A.M.Z. Slawin, S.P. Nolan, Organometallics. 32 (2013) 6265-6270.

[13] A. Włodarska, A. Kozioł, M. Dranka, A. Gryff-Keller, P. Szczeciński, J. Jurkowski, A. Pietrzykowski, Organometallics. 34 (2015) 577-581.

[14] W. Buchowicz, Ł. Banach, J. Conder, P.A. Guńka, D. Kubicki, P. Buchalski, Dalton Trans. 43 (2014) 5847-5857.

[15] M. Gholinejad, H.R. Shahsavari, M. Razeghi, M. Niazi, F. Hamed, J. Organomet. Chem. 796 (2015) 3-10.

[16] (a) S. Meiries, K. Speck, D.B. Cordes, A.M.Z. Slawin, S.P. Nolan, Organometallics. 32 (2013) 330-339. (b) S. Budagumpi, R.A. Haque, A.W. Salman, Coord. Chem. Rev. 256 (2012) 1787-1830. (c) G.C. Fortman, S.P. Nolan, Chem. Soc. Rev. 40 (2011) 5151-5169.

[17] (a) R. Visbal, A. Laguna, M.C. Gimeno, Chem. Comm. 49 (2013) 5642-5644. (b) J.C.Y. Lin, R.T.W. Huang, C.S. Lee, A. Bhattacharyya, W.S. Hwang, I.J.B. Lin, Chem. Rev. 109 (2009) 3561-3598. (c) M. Pellei, V. Gandin, M. Marinelli, C. Marzano, M. Yousufuddin, H.V.R. Dias, C. Santini, Inorg. Chem. 51 (2012) 98739882.

[18] E. Schuh, C. Pflüger, A. Citta, A. Folda, M.P. Rigobello, A. Bindoli, A. Casini, F. Mohr, J. Med. Chem. 55 (2012) 5518-5528.

[19] A.M. Oertel, V. Ritleng, M.J. Chetcuti, Organometallics. 31 (2012) 2829-2840.

[20] (a) M. Henrion, V. Ritleng, M.J. Chetcuti, ACS Catal. 5 (2015) 1283-1302. (b) C.-C. Lee, W-C. Ke, K-T. Chan, C-L. Lai, C-H. Hu, H.M. Lee, Chem. Eur. J. 13 (2007) 582-591. (c) J. Wu, A. Nova, D. Balcells, G.W. Brudvig, W. Dai, L.M. Guard, N. Hazari, P-H. Lin, R. Pokhrel, M.K. Takase, Chem. Eur. J. 20 (2014) 5327-5337.

[21] A.M. Oertel, J. Freudenreich, J. Gein, V. Ritleng, L.F. Veiros, M.J. Chetcuti, Organometallics. 30 (2011) 3400-3411.

[22] (a) O.R. Luca, D.L. Huang, M.K. Takase, R.H. Crabtree, New. J. Chem. 37 (2013) 3402-3405. (b) W. Buchowicz, W.Wojtczak, A. Pietrzykowski, A. Lupa, L.B. Jerzykiewicz, A. Makal, K. Woźniak, Eur. J. Inorg. Chem. (2010) 648-656. 
[23] C.D. Abernethy, A.H. Cowly, R.A. Jones, J. Organomet. Chem. 596 (2000) 3-5.

[24] K. Matsubara, K. Ueno, Y. Shibata, Organometallics. 25 (2006) 3422-3427.

[25] E.A. Standley, S.J. Smith, P. Müller, T.F. Jamison, Organometallics. 33 (2014) 20122018.

[26] N. Debono, A. Labande, E. Manoury, J-C. Daran, R. Poli, Organometallics. 29 (2010) 1879-1882.

[27] D.A. Malyshev, N.M. Scott, N. Marion, E.D. Stevens, V.P. Ananikov, I.P. Beletskaya, S.P. Nolan, Organometallics. 25 (2006) 4462-4470.

[28] (a) R.J. Errington, Advanced Practical Inorganic and Metalorganic Chemistry, Blackie Academic \& Professional, London, 1997. (b) D.F. Shriver, M.A. Drezdzon, The Manipulation of Air-Sensitive Compounds, 2nd ed., Wiley, New York, 1986.

[29] APEX2 (including SAINT and SADABS), Bruker AXS Inc., Madison, WI, 2012.

[30] G.M. Sheldrick, SHELXL96, Program for the Refinement of Crystal Structures, University of Göttingen, Germany, 1996.

[31] G.M. Sheldrick, Acta Cryst. A64 (2008) 112.

[32] O.V. Dolomanov, Bourhis, L.J., Gildea, R.J., Howard, J.A.K., Puschmann, H., J. Appl. Cryst., 2009, 42, 339., J. Appl. Cryst. 42 (2009) 339-341.

[33] V. Ritleng, A.M. Oertel, M.J. Chetchuti, Dalton Trans. 39 (2010) 8153-8160.

[34] H.M. Lee, P.L. Chiu, J.Y. Zeng, Inorg. Chim. Acta. 357 (2004) 4313-4321. 